\title{
Fractional Birkhoffian Mechanics Based on Quasi-Fractional Dynamics Models and Its Noether Symmetry
}

\author{
Yun-Die Jia ${ }^{1}$ and Yi Zhang $\mathbb{D}^{2}$ \\ ${ }^{1}$ College of Mathematical Sciences, Suzhou University of Science and Technology, Suzhou 215009, China \\ ${ }^{2}$ College of Civil Engineering, Suzhou University of Science and Technology, Suzhou 215011, China \\ Correspondence should be addressed to Yi Zhang; weidiezh@gmail.com
}

Received 14 December 2020; Revised 18 February 2021; Accepted 23 February 2021; Published 27 April 2021

Academic Editor: Gilberto Espinosa-Paredes

Copyright $\odot 2021$ Yun-Die Jia and Yi Zhang. This is an open access article distributed under the Creative Commons Attribution License, which permits unrestricted use, distribution, and reproduction in any medium, provided the original work is properly cited.

\begin{abstract}
This paper focuses on the exploration of fractional Birkhoffian mechanics and its fractional Noether theorems under quasifractional dynamics models. The quasi-fractional dynamics models under study are nonconservative dynamics models proposed by El-Nabulsi, including three cases: extended by Riemann-Liouville fractional integral (abbreviated as ERLFI), extended by exponential fractional integral (abbreviated as EEFI), and extended by periodic fractional integral (abbreviated as EPFI). First, the fractional Pfaff-Birkhoff principles based on quasi-fractional dynamics models are proposed, in which the Pfaff action contains the fractional-order derivative terms, and the corresponding fractional Birkhoff's equations are obtained. Second, the Noether symmetries and conservation laws of the systems are studied. Finally, three concrete examples are given to demonstrate the validity of the results.
\end{abstract}

\section{Introduction}

Symmetry theory plays an important role in mathematics, physics, and mechanics, and the study of symmetry properties of dynamic systems has become a very effective method to solve some practical problems. The most important and common symmetries are mainly of two kinds, namely, Noether symmetry and Lie symmetry. Noether's symmetry theory originated in 1918 and was first put forward by the famous mathematician Emmy Noether [1]. In this method, the relationship between symmetry and conserved quantity was established by using the invariance of Hamilton action under the infinitesimal group transformation of time and generalized coordinates. Candotti [2] and Desloge [3] applied Noether's theorem to classical mechanics. Djukić [4] established Noether's theorem for nonconservative systems. Liu [5] generalized Noether's theorem to nonholonomic mechanical systems. In 1979, Lutzky [6] applied the Lie method [7] of invariance of differential equations under infinitesimal group transformations to differential equations of motion for dynamical systems and started the study of Lie symmetry and conserved quantity of mechanical systems. Ibragimov [8] and Bluman [9] elaborated the role of Lie algebra and Lie group in studying the invariance of differential equations. Zhao [10] extended Lie symmetry theory to nonconservative mechanical systems. Mei [11, 12] systematically studied Noether symmetry, Lie symmetry of constrained mechanical systems, and corresponding conserved quantities. Recently, some new progress has been made in the study of these two symmetries (cf. [13-24] and references therein).

Fractional calculus is an important mathematical tool in science and engineering [25-28]. In recent decades, the research of fractional calculus has developed greatly, and its application fields have expanded to automatic control, quantum mechanics, and mechanical systems [29-35]. Riewe $[36,37]$ introduced the fractional variational problem for the first time in the study of nonconservative mechanics. In 2005, El-Nabulsi established a dynamical model of nonconservative systems under the framework of fractional calculus [38] based on the definition of Riemann-Liouville fractional integral (ERLFI). El-Nabulsi expanded the idea of 
dynamics modeling and successively put forward the dynamical models of nonconservative systems, which are extended by exponentially fractional integral (EEFI) and extended by periodic laws fractional integral (EPFI) $[39,40]$, respectively. The equations obtained from quasi-fractional dynamics models are similar to dynamical equations of classical conservative systems, which contain the generalized fractional external forces corresponding to dissipative forces, but the term with the fractional derivative does not show up. Different from other models, the fractional time integration of quasi-fractional dynamics models only needs one parameter. In this way, it simplifies the calculation of complex fractional calculus and provides a modeling method for nonconservative systems. Therefore, the quasi-fractional dynamics models can be used to study complex dynamical systems more conveniently. Frederico and Torres [41] first presented fractional Noether's theorems. Since then, studies on fractional Noether symmetry and conservation laws have been extensively developed [42-49]. In addition, Torres and Frederico studied Noether's theorems of fractional action-like variation problems $[50,51]$. In recent years, nonconservative dynamical systems based on quasi-fractional dynamical models have been studied deeply, and the corresponding dynamical equations and Noether conservation laws have been obtained [52-55]. However, most of the previous studies on the variational problems of quasi-fractional dynamics models are confined to Lagrangian framework and Hamiltonian framework.

It is well known that Birkhoffian mechanics is a new stage in the development of Hamiltonian mechanics [56-58]. Under canonical transformation, Hamilton canonical equation remains unchanged, but under general noncanonical transformation, it becomes Birkhoff's equation. Santilli [57] and Mei [58] both pointed out that Birkhoffian mechanics is the most general possible mechanics, which can be applied to hadron physics, space mechanics, statistical mechanics, biophysics, engineering, and other fields. Zhang and Zhai [59] has proposed the fractional Pfaff-Birkhoff principle and fractional Birkhoff's equations and proved that the fractional Hamilton principle is the special case of the fractional Pfaff-Birkhoff principle, and the fractional Hamilton equations and the fractional Lagrange equations are the special cases of the fractional Birkhoff's equations. Zhang and Zhou [52] proposed the quasi-fractional Pfaff-Birkhoff principle and derived corresponding quasi-fractional Birkhoff's equations which is based on the quasi-fractional model given by [38]. Up to now, some results have been obtained on Noether symmetry of fractional or quasi-fractional Birkhoffian systems, such as $[52,59-66]$. However, the results of these quasi-fractional Birkhoffian systems are limited to the Pfaff action containing only integral-order derivative terms. Here, we will further extend fractional Birkhoffian mechanics on the basis of three quasi-fractional dynamical models given in [38-40], where for the Pfaff actions, we consider contain fractional-order derivative terms. The quasi-fractional Lagrangian system and quasi-fractional Hamiltonian system are special cases of the results presented in this paper.
The text is organized as follows. In Section 2, the fractional Pfaff-Birkhoff principles under quasi-fractional dynamics models are presented and Birkhoff's equations are given, and nonisochronous variational formulae of the Pfaff action are driven. In Section 3, fractional Noether symmetries are well defined and their criteria are established. In Section 4, fractional Noether theorems are proved. For illustrating the application of the methods and results in this text, three examples are given in Section 5. In Section 6, we come to the conclusions.

\section{Fractional Birkhoff's Equations and Variation of Fractional Pfaff Action under Quasi-Fractional Dynamics Models}

For an introduction to fractional calculus and its basic theory, please refer to the monographs $[27,28]$.

2.1. Fractional Birkhoffian System Based on ERLFI. We consider a fractional Birkhoffian system determined by Birkhoff's variables $a^{\mu}(\mu=1,2, \ldots, 2 n)$, whose Birkhoff's functions are $R_{\mu}=R_{\mu}\left(\tau, a^{\nu}\right)$, the Birkhoffian is $B=B\left(\tau, a^{\nu}\right)$, $\beta$ is the order of fractional derivative, and $0 \leq \beta<1$.

Under the model of ERLFI, we define the Pfaff action as

$$
S_{R}=\frac{1}{\Gamma(\alpha)} \int_{a}^{b}\left[R_{\mu}\left(\tau, a^{\nu}\right)_{a} D_{\tau}^{\beta} a^{\mu}-B\left(\tau, a^{\nu}\right)\right](t-\tau)^{\alpha-1} \mathrm{~d} \tau,
$$

where ${ }_{a} D_{\tau}^{\beta} a^{\mu}(\mu=1,2, \ldots, 2 n)$ is the fractional derivative term.

The variational principle,

$$
\delta S_{R}=0,
$$

with commutative relation,

$$
\delta_{a} D_{\tau}^{\beta} a^{\mu}={ }_{a} D_{\tau}^{\beta} \delta a^{\mu},
$$

and boundary conditions,

$$
\begin{aligned}
\left.a^{\mu}\right|_{\tau=a} & =a_{1}^{\mu}, \\
\left.a^{\mu}\right|_{\tau=b} & =a_{2}^{\mu},
\end{aligned}
$$

is called the fractional Pfaff-Birkhoff principle based on ERLFI.

According to principle (2), we drive

$$
\begin{array}{r}
\left(\frac{\partial R_{v}}{\partial a^{\mu}} D_{\tau}^{\beta} a^{\nu}-\frac{\partial B}{\partial a^{\mu}}\right)(t-\tau)^{\alpha-1}+{ }_{\tau} D_{b}^{\beta}\left[R_{\mu}(t-\tau)^{\alpha-1}\right]=0, \\
(\mu=1,2, \ldots, 2 n) .
\end{array}
$$

Equation (5) is the fractional Birkhoff's equations based on ERLFI.

If $\beta \longrightarrow 1$, equation (5) becomes Birkhoff's equations based on ERLFI. If $\beta \longrightarrow 1$ and $\alpha \longrightarrow 1$, equation (5) becomes classical Birkhoff's equations [58].

Take the infinitesimal transformations: 


$$
\begin{aligned}
\bar{\tau} & =\tau+\Delta \tau, \\
\bar{a}^{\mu}(\bar{\tau}) & =a^{\mu}(\tau)+\Delta a^{\mu}, \quad(\mu=1,2, \ldots, 2 n),
\end{aligned}
$$

and their first-order extensions

$$
\begin{aligned}
\bar{\tau} & =\tau+\varepsilon_{\sigma} \xi_{0}^{\sigma}\left(\tau, a^{\nu}\right), \\
\bar{a}^{\mu}(\bar{\tau}) & =a^{\mu}(\tau)+\varepsilon_{\sigma} \xi_{\mu}^{\sigma}\left(\tau, a^{\nu}\right), \quad(\mu=1,2, \ldots, 2 n),
\end{aligned}
$$

where $\varepsilon_{\sigma}$ is the infinitesimal parameter and $\xi_{0}^{\sigma}$ and $\xi_{\mu}^{\sigma}$ are the generating functions.

Under transformation (6), the Pfaff action (1) is transformed into

$$
S_{R}(\bar{\gamma})=\frac{1}{\Gamma(\alpha)} \int_{\bar{a}}^{\bar{b}}\left[R_{\mu}\left(\bar{\tau}, \bar{a}^{\nu}\right)_{\bar{a}} D_{\bar{\tau}}^{\beta} \bar{a}^{\mu}-B\left(\bar{\tau}, \bar{a}^{\nu}\right)\right](t-\bar{\tau})^{\alpha-1} \mathrm{~d} \bar{\tau} .
$$

And, we have

$$
\begin{aligned}
& S_{R}(\bar{\gamma})-S_{R}(\gamma) \\
& =\frac{1}{\Gamma(\alpha)} \int_{\bar{a}}^{\bar{b}}\left[R_{\mu}\left(\bar{\tau}, \bar{a}^{\nu}\right)_{\bar{a}} D_{\bar{\tau}}^{\beta} \bar{a}^{\mu}-B\left(\bar{\tau}, \bar{a}^{\nu}\right)\right] \\
& (t-\bar{\tau})^{\alpha-1} \mathrm{~d} \bar{\tau} \\
& -\frac{1}{\Gamma(\alpha)} \int_{a}^{b}\left[R_{\mu}\left(\tau, a^{\nu}\right)_{a} D_{\tau}^{\beta} a^{\mu}-B\left(\tau, a^{\nu}\right)\right] \\
& (t-\tau)^{\alpha-1} \mathrm{~d} \tau \\
& =\frac{1}{\Gamma(\alpha)} \int_{a}^{b}\left\{\left[R_{\mu}\left(\tau+\Delta \tau, a^{\nu}+\Delta a^{\nu}\right)\right]\right\} \\
& \left({ }_{a} D_{\tau}^{\beta} a^{\mu}+{ }_{a} D_{\tau}^{\beta} \Delta a^{\mu}-{ }_{a} D_{\tau}^{\beta}\left(\dot{a}^{\mu} \Delta \tau\right)+\Delta \tau{ }_{a} D_{\tau}^{\beta} \dot{a}^{\mu}\right) \\
& \left.-B\left(\tau+\Delta \tau, a^{\nu}+\Delta a^{\nu}\right)\right] \\
& (t-(\tau+\Delta \tau))^{\alpha-1}\left(1+\frac{\mathrm{d}}{\mathrm{d} \tau} \Delta \tau\right) \\
& \left.-\left[R_{\mu}\left(\tau, a^{\nu}\right)_{a} D_{\tau}^{\beta} a^{\mu}-B\left(\tau, a^{\nu}\right)\right](t-\tau)^{\alpha-1}\right\} \mathrm{d} \tau .
\end{aligned}
$$

Let $\Delta S_{R}$ be nonisochronous variation of $S_{R}$, which is the main line part of $S_{R}(\bar{\gamma})-S_{R}(\gamma)$ relative to $\varepsilon$, and we obtain

$$
\begin{aligned}
\Delta S_{R}= & \frac{1}{\Gamma(\alpha)} \int_{a}^{b}\left\{\left(\frac{\partial R_{\mu}}{\partial a^{\nu} a} D_{\tau}^{\beta} a^{\mu}-\frac{\partial B}{\partial a^{\nu}}\right)(t-\tau)^{\alpha-1} \Delta a^{\nu}\right\} \\
& +\left(\frac{\partial R_{\mu}}{\partial \tau} D^{\beta} D_{\tau}^{\beta} a^{\mu}-\frac{\partial B}{\partial \tau}\right)(t-\tau)^{\alpha-1} \Delta \tau \\
& +\left(R_{\mu a} D_{\tau}^{\beta} a^{\mu}-B\right)(t-\tau)^{\alpha-1} \frac{\mathrm{d}}{\mathrm{d} \tau} \Delta \tau \\
& +R_{\mu}\left({ }_{a} D_{\tau}^{\beta} \Delta a^{\mu}-{ }_{a} D_{\tau}^{\beta}\left(\dot{a}^{\mu} \Delta \tau\right)+\Delta \tau_{a} D_{\tau}^{\beta} \dot{a}^{\mu}\right)(t-\tau)^{\alpha-1}
\end{aligned}
$$$$
\left.-\left(R_{\mu a} D_{\tau}^{\beta} a^{\mu}-B\right)(\alpha-1)(t-\tau)^{\alpha-2} \Delta \tau\right\} \mathrm{d} \tau
$$

Since

$$
\begin{aligned}
\delta a^{\mu} & =\Delta a^{\mu}-\dot{a}^{\mu} \Delta \tau, \\
\Delta \dot{a}^{\mu} & =\frac{\mathrm{d}}{\mathrm{d} \tau}\left(\Delta a^{\mu}\right)-\dot{a}^{\mu} \frac{\mathrm{d}}{\mathrm{d} \tau}(\Delta \tau),
\end{aligned}
$$

then we obtain

$$
\begin{aligned}
\Delta S_{R}= & \frac{1}{\Gamma(\alpha)} \int_{a}^{b}\left\{\frac { \mathrm { d } } { \mathrm { d } \tau } \left[\left(R_{\mu a} D_{\tau}^{\beta} a^{\mu}-B\right)(t-\tau)^{\alpha-1} \Delta \tau\right.\right. \\
& \left.+\int_{a}^{\tau}\left(R_{\mu a} D_{\tau}^{\beta} \delta a^{\mu}(t-s)^{\alpha-1}-\delta a^{\mu}{ }_{s} D_{b}^{\beta}\left(R_{\mu}(t-s)^{\alpha-1}\right)\right) \mathrm{d} s\right] \\
& \left.+\left[\left(\frac{\partial R_{\nu}}{\partial a^{\mu} a} D_{\tau}^{\beta} a^{\nu}-\frac{\partial B}{\partial a^{\mu}}\right)(t-\tau)^{\alpha-1}+{ }_{\tau} D_{b}^{\beta}\left(R_{\mu}(t-\tau)^{\alpha-1}\right)\right] \delta a^{\mu}\right\} \mathrm{d} \tau .
\end{aligned}
$$

By using formula (7), we obtain

$$
\begin{aligned}
\Delta S_{R}= & \frac{1}{\Gamma(\alpha)} \int_{a}^{b} \varepsilon_{\sigma}\left\{\frac { \mathrm { d } } { \mathrm { d } \tau } \left[\left(R_{\mu a} D_{\tau}^{\beta} a^{\mu}-B\right)(t-\tau)^{\alpha-1} \xi_{0}^{\sigma}\right.\right. \\
& \left.+\int_{a}^{\tau}\left(R_{\mu a} D_{s}^{\beta}\left(\xi_{\mu}^{\sigma}-\dot{a}^{\mu} \xi_{0}^{\sigma}\right)(t-s)^{\alpha-1}-\left(\xi_{\mu}^{\sigma}-\dot{a}^{\mu} \xi_{0}^{\sigma}\right)_{s} D_{b}^{\beta}\left(R_{\mu}(t-s)^{\alpha-1}\right)\right) \mathrm{d} s\right] \\
& \left.+\left[\left(\frac{\partial R_{v}}{\partial a^{\mu} a} D_{\tau}^{\beta} a^{\nu}-\frac{\partial B}{\partial a^{\mu}}\right)(t-\tau)^{\alpha-1}+{ }_{\tau} D_{b}^{\beta}\left(R_{\mu}(t-\tau)^{\alpha-1}\right)\right]\left(\xi_{\mu}^{\sigma}-\dot{a}^{\mu} \xi_{0}^{\sigma}\right)\right\} \mathrm{d} \tau .
\end{aligned}
$$


Equations (10) and (13) are two mutually equivalent formulas derived from Pfaff action (1).

2.2. Fractional Birkhoffian System Based on EEFI. Under the model of EEFI, we define the Pfaff action as

$S_{E}=\frac{1}{\Gamma(\alpha)} \int_{a}^{b}\left[R_{\mu}\left(\tau, a^{\nu}\right)_{a} D_{\tau}^{\beta} a^{\mu}-B\left(\tau, a^{\nu}\right)\right](\cosh t-\cosh \tau)^{\alpha-1} \mathrm{~d} \tau$.

The fractional Pfaff-Birkhoff principle is

$$
\delta S_{E}=0,
$$

under commutative relation,

$$
\delta_{a} D_{\tau}^{\beta} a^{\mu}={ }_{a} D_{\tau}^{\beta} \delta a^{\mu},
$$

and boundary conditions,

$$
\begin{aligned}
& \left.a^{\mu}\right|_{\tau=a}=a_{1}^{\mu}, \\
& \left.a^{\mu}\right|_{\tau=b}=a_{2}^{\mu} .
\end{aligned}
$$

The fractional Birkhoff's equations are

$$
\begin{aligned}
& \left(\frac{\partial R_{v}}{\partial a^{\mu} a} D_{\tau}^{\beta} a^{\nu}-\frac{\partial B}{\partial a^{\mu}}\right)(\cosh t-\cosh \tau)^{\alpha-1} \\
& \quad+{ }_{\tau} D_{b}^{\beta}\left[R_{\mu}(\cosh t-\cosh \tau)^{\alpha-1}\right]=0, \quad(\mu=1,2, \ldots, 2 n) .
\end{aligned}
$$

If $\beta \longrightarrow 1$, equation (18) becomes Birkhoff's equations based on EEFI. If $\beta \longrightarrow 1$ and $\alpha \longrightarrow 1$, equation (18) becomes classical Birkhoff's equations [58].

According to formula (6), action (14) is transformed into

$$
S_{E}(\bar{\gamma})=\frac{1}{\Gamma(\alpha)} \int_{\bar{a}}^{\bar{b}}\left[R_{\mu}\left(\bar{\tau}, \bar{a}^{\nu}\right)_{\bar{a}} D_{\bar{\tau}}^{\beta} \bar{a}^{\mu}-B\left(\bar{\tau}, \bar{a}^{\nu}\right)\right](\cosh t-\cosh \bar{\tau})^{\alpha-1} \mathrm{~d} \bar{\tau},
$$

and we have

$$
\begin{aligned}
S_{E} & (\bar{\gamma})-S_{E}(\gamma) \\
& =\frac{1}{\Gamma(\alpha)} \int_{\bar{a}}^{\bar{b}}\left[R_{\mu}\left(\bar{\tau}, \bar{a}^{\nu}\right)_{\bar{a}} D_{\bar{\tau}}^{\beta} \bar{a}^{\mu}-B\left(\bar{\tau}, \bar{a}^{\nu}\right)\right] \\
& (\cosh t-\cosh \bar{\tau})^{\alpha-1} \mathrm{~d} \bar{\tau} \\
& -\frac{1}{\Gamma(\alpha)} \int_{a}^{b}\left[R_{\mu}\left(\tau, a^{\nu}\right)_{a} D_{\tau}^{\beta} a^{\mu}-B\left(\tau, a^{\nu}\right)\right] \\
& (\cosh t-\cosh \tau)^{\alpha-1} \mathrm{~d} \tau \\
& =\frac{1}{\Gamma(\alpha)} \int_{a}^{b}\left\{\left[R_{\mu}\left(\tau+\Delta \tau, a^{\nu}+\Delta a^{\nu}\right)\right]\right\} \\
& \left({ }_{a} D_{\tau}^{\beta} a^{\mu}+{ }_{a} D_{\tau}^{\beta} \Delta a^{\mu}-{ }_{a} D_{\tau}^{\beta}\left(\dot{a}^{\mu} \Delta \tau\right)+\Delta \tau D^{\beta} D_{\tau} \dot{a}^{\mu}\right) \\
& \left.-B\left(\tau+\Delta \tau, a^{\nu}+\Delta a^{\nu}\right)\right] \\
& (\cosh t-\cosh (\tau+\Delta \tau))^{\alpha-1}\left(1+\frac{\mathrm{d}}{\mathrm{d} \tau} \Delta \tau\right) \\
& \left.-\left[R_{\mu}\left(\tau, a^{\nu}\right)_{a} D_{\tau}^{\beta} a^{\mu}-B\left(\tau, a^{\nu}\right)\right](\cosh t-\cosh \tau)^{\alpha-1}\right\} \mathrm{d} \tau .
\end{aligned}
$$

So, the nonisochronous variation $\Delta S_{E}$ of action $S_{E}$ is

$$
\begin{aligned}
\Delta S_{E}= & \frac{1}{\Gamma \alpha} \\
& \cdot \int_{a}^{b}\left\{\frac{\partial R_{\mu}}{\partial a^{\nu}} D_{\tau}^{\beta} a^{\mu}-\frac{\partial B}{\partial a^{\nu}} \cos h t-\cos h \tau^{\alpha-1} \Delta a^{\nu}\right. \\
& +\frac{\partial R_{\mu}}{\partial \tau}{ } D_{\tau}^{\beta} a^{\mu}-\frac{\partial B}{\partial \tau} \cos h t-\cos h \tau^{\alpha-1} \Delta \tau \\
& +R_{\mu a} D_{\tau}^{\beta} a^{\mu}-B \cos h t-\cos h \tau^{\alpha-1} \frac{\mathrm{d}}{\mathrm{d} \tau} \Delta \tau \\
& +R_{\mu a} D_{\tau}^{\beta} \Delta a^{\mu}-{ }_{a} D_{\tau}^{\beta} \dot{a}^{\mu} \Delta \tau+\Delta \tau_{a} D_{\tau}^{\beta} \dot{a}^{\mu} \cos h t-\cos h \tau^{\alpha-1} \\
& \left.+\alpha-1 \sin h \tau \cos h t-\cos h \tau^{\alpha-2} \Delta \tau\right\} \mathrm{d} \tau .
\end{aligned}
$$

Equation (21) can also be written as

$$
\begin{aligned}
\Delta S_{E}= & \frac{1}{\Gamma \alpha} \\
& \cdot \int_{a}^{b}\left\{\frac { d } { d \tau } \left[R_{\mu a} D_{\tau}^{\beta} a^{\mu}-B \cosh t-\cosh \tau^{\alpha-1} \Delta \tau\right.\right. \\
& +\int_{a}^{\tau} R_{\mu a} D_{\tau}^{\beta} \delta a^{\mu} \cosh t-\cosh s^{\alpha-1} \\
& \left.-\delta a^{\mu}{ }_{s} D_{b}^{\beta} R_{\mu} \cosh t-\cosh s^{\alpha-1} \mathrm{~d} s\right] \\
& +\left[\frac{\partial R_{v}}{\partial a^{\mu} D^{\beta}}{ }_{\tau} a^{\nu}-\frac{\partial B}{\partial a^{\mu}} \cos h t-\cos h \tau^{\alpha-1}\right. \\
& \left.\left.+{ }_{\tau} D_{b}^{\beta} R_{\mu} \cosh t-\cosh \tau^{\alpha-1}\right] \delta a^{\mu}\right\} \mathrm{d} \tau .
\end{aligned}
$$

By using formula (7), we have

$$
\begin{aligned}
& \Delta S_{E}=\frac{1}{\Gamma \alpha} \\
& \cdot \int_{a}^{b} \varepsilon_{\sigma}\left\{\frac { d } { d \tau } \left[R_{\mu a} D_{\tau}^{\beta} a^{\mu}-B \cosh t-\cosh \tau^{\alpha-1} \xi_{0}^{\sigma}\right.\right. \\
& +\int_{a}^{\tau} R_{\mu a} D_{s}^{\beta} \xi_{\mu}^{\sigma}-\dot{a}^{\mu} \xi_{0}^{\sigma} \cosh t-\cosh s^{\alpha-1} \\
& \left.-\xi_{\mu}^{\sigma}-\dot{a}^{\mu} \xi_{0 s}^{\sigma} D_{b}^{\beta} R_{\mu} \cosh t-\cosh s^{\alpha-1} \mathrm{~d} s\right] \\
& +\left[\frac{\partial R_{v}}{\partial a^{\mu} a} D_{\tau}^{\beta} a^{v}-\frac{\partial B}{\partial a^{\mu}} \cosh t-\cosh \tau^{\alpha-1}\right. \\
& \left.\left.{ }_{\tau} D_{b}^{\beta} R_{\mu} \cosh t-\cosh \tau^{\alpha-1}\right] \xi_{\mu}^{\sigma}-\dot{a}^{\mu} \xi_{0}^{\sigma}\right\} \mathrm{d} \tau .
\end{aligned}
$$

Equations (21) and (23) are two mutually equivalent formulas derived from Pfaff action (14). 
2.3. Fractional Birkhoffian System Based on EPFI. Under the model of EPFI, we define the Pfaff action as

$$
\begin{aligned}
S_{P}= & \frac{1}{\Gamma(\alpha)} \int_{a}^{b}\left\{\left[R_{\mu}\left(\tau, a^{\nu}\right)_{a} D_{\tau}^{\beta} a^{\mu}-B\left(\tau, a^{\nu}\right)\right] \sin \right. \\
& \left.\cdot\left((\alpha-1)(t-\tau)+\frac{\pi}{2}\right)\right\} \mathrm{d} \tau .
\end{aligned}
$$

The fractional Pfaff-Birkhoff principle is

$$
\delta S_{P}=0,
$$

under commutative relation,

$$
\delta_{a} D_{\tau}^{\beta} a^{\mu}={ }_{a} D_{\tau}^{\beta} \delta a^{\mu}
$$

and boundary conditions,

$$
\begin{aligned}
& \left.a^{\mu}\right|_{\tau=a}=a_{1}^{\mu}, \\
& \left.a^{\mu}\right|_{\tau=b}=a_{2}^{\mu} .
\end{aligned}
$$

The fractional Birkhoff's equations are

$$
\begin{aligned}
& \left(\frac{\partial R_{v}}{\partial a^{\mu}} D_{\tau}^{\beta} a^{\nu}-\frac{\partial B}{\partial a^{\mu}}\right) \sin \left((\alpha-1)(t-\tau)+\frac{\pi}{2}\right) \\
& \quad+{ }_{\tau} D_{b}^{\beta}\left[R_{\mu} \sin \left((\alpha-1)(t-\tau)+\frac{\pi}{2}\right)\right]=0, \quad(\mu=1,2, \ldots, 2 n) .
\end{aligned}
$$

If $\beta \longrightarrow 1$, equation (28) becomes Birkhoff's equations based on EPFI. If $\beta \longrightarrow 1$ and $\alpha \longrightarrow 1$, equation (28) becomes the classical Birkhoff's equations [58].

According to formula (6), action (24) is transformed into

$$
\begin{aligned}
S_{P}(\bar{\gamma})= & \frac{1}{\Gamma(\alpha)} \int_{\bar{a}}^{\bar{b}}\left[R_{\mu}\left(\bar{\tau}, \bar{a}^{\nu}\right)_{\bar{a}} D_{\bar{\tau}}^{\beta} \bar{a}^{\mu}-B\left(\bar{\tau}, \bar{a}^{\nu}\right)\right] \sin \\
& \cdot\left((\alpha-1)(t-\bar{\tau})+\frac{\pi}{2}\right) d \bar{\tau},
\end{aligned}
$$

and we have

$$
S_{P}(\bar{\gamma})-S_{P}(\gamma)
$$

$$
\begin{aligned}
& =\frac{1}{\Gamma(\alpha)} \int_{\bar{a}}^{\bar{b}}\left[R_{\mu}\left(\bar{\tau}, \bar{a}^{\nu}\right)_{\bar{a}} D_{\bar{\tau}}^{\beta} \bar{a}^{\mu}-B\left(\bar{\tau}, \bar{a}^{\nu}\right)\right] \\
& \sin \left((\alpha-1)(t-\bar{\tau})+\frac{\pi}{2}\right) \mathrm{d} \bar{\tau}
\end{aligned}
$$$$
-\frac{1}{\Gamma(\alpha)} \int_{a}^{b}\left[R_{\mu}\left(\tau, a^{\nu}\right)_{a} D_{\tau}^{\beta} a^{\mu}-B\left(\tau, a^{\nu}\right)\right]
$$$$
\sin \left((\alpha-1)(t-\tau)+\frac{\pi}{2}\right) \mathrm{d} \tau
$$$$
=\frac{1}{\Gamma(\alpha)} \int_{a}^{b}\left\{\left[R_{\mu}\left(\tau+\Delta \tau, a^{\nu}+\Delta a^{\nu}\right)\right]\right\}
$$$$
\left({ }_{a} D_{\tau}^{\beta} a^{\mu}+{ }_{a} D_{\tau}^{\beta} \Delta a^{\mu}\right)
$$

$\left(-{ }_{a} D_{\tau}^{\beta}\left(\dot{a}^{\mu} \Delta \tau\right)+\Delta \tau_{a} D_{\tau}^{\beta} \dot{a}^{\mu}\right)$

$$
\left.-B\left(\tau+\Delta \tau, a^{v}+\Delta a^{\nu}\right)\right]
$$

$$
\begin{aligned}
& \sin \left((\alpha-1)(t-(\tau+\Delta \tau))+\frac{\pi}{2}\right) \\
& \left(1+\frac{\mathrm{d}}{\mathrm{d} \tau} \Delta \tau\right) \\
& -\left[R_{\mu}\left(\tau, a^{\nu}\right)_{a} D_{\tau}^{\beta} a^{\mu}-B\left(\tau, a^{\nu}\right)\right] \\
& \left.\sin \left((\alpha-1)(t-\tau)+\frac{\pi}{2}\right)\right\} \mathrm{d} \tau .
\end{aligned}
$$

So, we have

$$
\begin{aligned}
\Delta S_{P}= & \frac{1}{\Gamma(\alpha)} \int_{a}^{b}\left\{\left(\frac{\partial R_{\mu}}{\partial a^{\nu} a} D_{\tau}^{\beta} a^{\mu}-\frac{\partial B}{\partial a^{\nu}}\right) \sin \left((\alpha-1)(t-\tau)+\frac{\pi}{2}\right) \Delta a^{\nu}+\left(\frac{\partial R_{\mu}}{\partial \tau} D_{a}^{\beta} a^{\mu}-\frac{\partial B}{\partial \tau}\right) \sin \left((\alpha-1)(t-\tau)+\frac{\pi}{2}\right) \Delta \tau\right. \\
& +\left(R_{\mu a} D_{\tau}^{\beta} a^{\mu}-B\right) \sin \left((\alpha-1)(t-\tau)+\frac{\pi}{2}\right) \frac{\mathrm{d}}{\mathrm{d} \tau} \Delta \tau+R_{\mu}\left({ }_{a} D_{\tau}^{\beta} \Delta a^{\mu}-{ }_{a} D_{\tau}^{\beta}\left(\dot{a}^{\mu} \Delta \tau\right)+\Delta \tau_{a} D_{\tau}^{\beta} \dot{a}^{\mu}\right) \sin \left((\alpha-1)(t-\tau)+\frac{\pi}{2}\right) \\
& \left.-(\alpha-1) \cos \left((\alpha-1)(t-\tau)+\frac{\pi}{2}\right)\right\} \mathrm{d} \tau .
\end{aligned}
$$


Equation (31) can also be written as

$$
\begin{aligned}
\Delta S_{P}= & \frac{1}{\Gamma(\alpha)} \int_{a}^{b}\left\{\frac { \mathrm { d } } { \mathrm { d } \tau } \left[\left(R_{\mu a} D_{\tau}^{\beta} a^{\mu}-B\right) \sin \left((\alpha-1)(t-\tau)+\frac{\pi}{2}\right) \Delta \tau+\int_{a}^{\tau}\left(R_{\mu a} D_{s}^{\beta} \delta a^{\mu} \sin \left((\alpha-1)(t-s)+\frac{\pi}{2}\right)\right.\right.\right. \\
& \left.\left.-\delta a^{\mu}{ }_{s} D_{b}^{\beta}\left(R_{\mu} \sin \left((\alpha-1)(t-s)+\frac{\pi}{2}\right)\right)\right) \mathrm{d} s\right]+\left[\left(\frac{\partial R_{v}}{\partial a^{\mu}} D_{\tau}^{\beta} a^{\nu}-\frac{\partial B}{\partial a^{\mu}}\right) \sin \left((\alpha-1)(t-\tau)+\frac{\pi}{2}\right)\right. \\
& \left.\left.+{ }_{\tau} D_{b}^{\beta}\left(R_{\mu} \sin \left((\alpha-1)(t-\tau)+\frac{\pi}{2}\right)\right)\right] \delta a^{\mu}\right\} \mathrm{d} \tau .
\end{aligned}
$$

By using formula (7), we have

$$
\begin{aligned}
\Delta S_{P}= & \frac{1}{\Gamma(\alpha)} \int_{a}^{b} \varepsilon_{\sigma}\left\{\frac { \mathrm { d } } { \mathrm { d } \tau } \left[\left(R_{\mu a} D_{\tau}^{\beta} a^{\mu}-B\right) \sin \left((\alpha-1)(t-\tau)+\frac{\pi}{2}\right) \xi_{0}^{\sigma}\right.\right. \\
& \left.+\int_{a}^{\tau}\left(R_{\mu a} D_{s}^{\beta}\left(\xi_{\mu}^{\sigma}-\dot{a}^{\mu} \xi_{0}^{\sigma}\right) \sin \left((\alpha-1)(t-s)+\frac{\pi}{2}\right)-\left(\xi_{\mu}^{\sigma}-\dot{a}^{\mu} \xi_{0}^{\sigma}\right)_{s} D_{b}^{\beta}\left(R_{\mu} \sin \left((\alpha-1)(t-s)+\frac{\pi}{2}\right)\right)\right)\right] \mathrm{d} s \\
& \left.+\left[\left(\frac{\partial R_{v}}{\partial a^{\mu} a} D_{\tau}^{\beta} a^{v}-\frac{\partial B}{\partial a^{\mu}}\right) \sin \left((\alpha-1)(t-\tau)+\frac{\pi}{2}\right)+{ }_{\tau} D_{b}^{\beta}\left(R_{\mu} \sin \left((\alpha-1)(t-\tau)+\frac{\pi}{2}\right)\right)\right]\left(\xi_{\mu}^{\sigma}-\dot{a}^{\mu} \xi_{0}^{\sigma}\right)\right\} \mathrm{d} \tau
\end{aligned}
$$

Equations (31) and (33) are two mutually equivalent formulas derived from Pfaff action (24).

\section{Fractional Noether Symmetries under Quasi- Fractional Dynamics Models}

Next, we will define the Noether symmetries of the system under three quasi-fractional dynamics models and establish their criteria.

3.1. Fractional Noether Symmetries Based on ERLFI
Definition 1. If the Pfaff action (1) satisfies the equality

$$
\Delta S_{R}=0
$$

then transformation (6) is said to be Noether symmetric for system (5).

According to Definition 1, using formulas (10) and (13), we have the following.

Criterion 1. If transformation (6) is Noether symmetric, then the equation,

$$
\begin{aligned}
& \left(\frac{\partial R_{\mu}}{\partial a^{\nu} a} D_{\tau}^{\beta} a^{\mu}-\frac{\partial B}{\partial a^{\nu}}\right) \Delta a^{\nu}+\left(\frac{\partial R_{\mu}}{\partial \tau}{ }_{a} D_{\tau}^{\beta} a^{\mu}-\frac{\partial B}{\partial \tau}\right) \Delta \tau+\left(R_{\mu a} D_{\tau}^{\beta} a^{\mu}-B\right) \frac{\mathrm{d}}{\mathrm{d} \tau} \Delta \tau \\
& \quad+R_{\mu}\left[{ }_{a} D_{\tau}^{\beta} \Delta a^{\mu}-{ }_{a} D_{\tau}^{\beta}\left(\dot{a}^{\mu} \Delta \tau\right)+{ }_{a} D_{\tau}^{\beta} \dot{a}^{\mu} \Delta \tau\right]-\left(R_{\mu a} D_{\tau}^{\beta} a^{\mu}-B\right) \frac{\alpha-1}{t-\tau} \Delta \tau=0
\end{aligned}
$$

needs to be satisfied.

$$
\begin{aligned}
& \left(\frac{\partial R_{\mu}}{\partial a^{\nu} a} D_{\tau}^{\beta} a^{\mu}-\frac{\partial B}{\partial a^{\nu}}\right) \xi_{\nu}^{\sigma}+\left(\frac{\partial R_{\mu}}{\partial \tau}{ }_{a} D_{\tau}^{\beta} a^{\mu}-\frac{\partial B}{\partial \tau}\right) \xi_{0}^{\sigma}+\left(R_{\mu a} D_{\tau}^{\beta} a^{\mu}-B\right) \dot{\xi}_{0}^{\sigma} \\
& \quad+R_{\mu}\left({ }_{a} D_{\tau}^{\beta} \xi_{\mu}^{\sigma}-{ }_{a} D_{\tau}^{\beta}\left(\dot{a}^{\mu} \xi_{0}^{\sigma}\right)+{ }_{a} D_{\tau}^{\beta} \dot{a}^{\mu} \xi_{0}^{\sigma}\right)-\left(R_{\mu a} D_{\tau}^{\beta} a^{\mu}-B\right) \frac{\alpha-1}{t-\tau} \xi_{0}^{\sigma}=0, \quad(\sigma=1,2, \ldots, r) .
\end{aligned}
$$


If $r=1$, equation (36) gives the fractional Noether identity based on ERLFI.
Criterion 2. If transformation (7) is Noether symmetric, then the following $r$ equations,

$$
\begin{aligned}
& \frac{\mathrm{d}}{\mathrm{d} \tau}\left\{\left(R_{\mu a} D_{\tau}^{\beta} a^{\mu}-B\right)(t-\tau)^{\alpha-1} \xi_{0}^{\sigma}+\int_{a}^{\tau}\left[R_{\mu a} D_{s}^{\beta}\left(\xi_{\mu}^{\sigma}-\dot{a}^{\mu} \xi_{0}^{\sigma}\right)(t-s)^{\alpha-1}-\left(\xi_{\mu}^{\sigma}-\dot{a}^{\mu} \xi_{0}^{\sigma}\right)_{s} D_{b}^{\beta}\left[R_{\mu}(t-s)^{\alpha-1}\right]\right] \mathrm{d} s\right\} \\
& \quad+\left[\left(\frac{\partial R_{v}}{\partial a^{\mu} a} D_{\tau}^{\beta} a^{\nu}-\frac{\partial B}{\partial a^{\mu}}\right)(t-\tau)^{\alpha-1}+{ }_{\tau} D_{b}^{\beta}\left(R_{\mu}(t-\tau)^{\alpha-1}\right)\right]\left(\xi_{\mu}^{\sigma}-\dot{a}^{\mu} \xi_{0}^{\sigma}\right)=0, \quad(\sigma=1,2, \ldots, r),
\end{aligned}
$$

need to be satisfied.

Definition 2. If the Pfaff action (1) satisfies the equality

$$
\Delta S_{R}=-\frac{1}{\Gamma(\alpha)} \int_{a}^{b} \frac{\mathrm{d}}{\mathrm{d} \tau}(\Delta G) \mathrm{d} \tau
$$

where $\Delta G=\varepsilon_{\sigma} G^{\sigma}$ and $G^{\sigma}=G^{\sigma}\left(\tau, a^{\nu}\right)$ is the gauge function, then transformation (6) is said to be Noether quasi-symmetric for system (5).
According to Definition 2, using formulas (10) and (13), we have the following.

Criterion 3. . If transformation (6) is Noether quasi-symmetric, then the equation,

$$
\begin{aligned}
& \left(\frac{\partial R_{\mu}}{\partial a^{\nu} a} D_{\tau}^{\beta} a^{\mu}-\frac{\partial B}{\partial a^{\nu}}\right) \Delta a^{\nu}+\left(\frac{\partial R_{\mu}}{\partial \tau}{ }_{a} D_{\tau}^{\beta} a^{\mu}-\frac{\partial B}{\partial \tau}\right) \Delta \tau \\
& +\left(R_{\mu a} D_{\tau}^{\beta} a^{\mu}-B\right) \frac{\mathrm{d}}{\mathrm{d} \tau} \Delta \tau+R_{\mu}\left[{ }_{a} D_{\tau}^{\beta} \Delta a^{\mu}-{ }_{a} D_{\tau}^{\beta}\left(\dot{a}^{\mu} \Delta \tau\right)+{ }_{a} D_{\tau}^{\beta} \dot{a}^{\mu} \Delta \tau\right] \\
& \quad-\left(R_{\mu a} D_{\tau}^{\beta} a^{\mu}-B\right) \frac{\alpha-1}{t-\tau} \Delta \tau=-\frac{\mathrm{d}}{\mathrm{d} \tau}(\Delta G)(t-\tau)^{1-\alpha}
\end{aligned}
$$

needs to be satisfied.

Equation (39) can be written as $r$ equations:

$$
\begin{aligned}
& \left(\frac{\partial R_{\mu}}{\partial a^{\nu} a} D_{\tau}^{\beta} a^{\mu}-\frac{\partial B}{\partial a^{\nu}}\right) \xi_{\nu}^{\sigma}+\left(\frac{\partial R_{\mu}}{\partial \tau}{ }_{a} D_{\tau}^{\beta} a^{\mu}-\frac{\partial B}{\partial \tau}\right) \xi_{0}^{\sigma} \\
& \quad+\left(R_{\mu a} D_{\tau}^{\beta} a^{\mu}-B\right) \dot{\xi}_{0}^{\sigma}+R_{\mu}\left({ }_{a} D_{\tau}^{\beta} \xi_{\mu}^{\sigma}-{ }_{a} D_{\tau}^{\beta}\left(\dot{a}^{\mu} \xi_{0}^{\sigma}\right)+{ }_{a} D_{\tau}^{\beta} \dot{a}^{\mu} \xi_{0}^{\sigma}\right) \\
& \quad-\left(R_{\mu a} D_{\tau}^{\beta} a^{\mu}-B\right) \frac{\alpha-1}{t-\tau} \xi_{0}^{\sigma}=-\dot{G}^{\sigma}(t-\tau)^{1-\alpha}, \quad(\sigma=1,2, \ldots, r)
\end{aligned}
$$

If $r=1$, equation (40) also gives the fractional Noether identity based on ERLFI.
Criterion 4. If transformation (7) is Noether quasi-symmetric, then the following $r$ equations,

$$
\begin{aligned}
& \frac{\mathrm{d}}{\mathrm{d} \tau}\left\{\left(R_{\mu a} D_{\tau}^{\beta} a^{\mu}-B\right)(t-\tau)^{\alpha-1} \xi_{0}^{\sigma}+\int_{a}^{\tau}\left[R_{\mu a} D_{s}^{\beta}\left(\xi_{\mu}^{\sigma}-\dot{a}^{\mu} \xi_{0}^{\sigma}\right)(t-s)^{\alpha-1}-\left(\xi_{\mu}^{\sigma}-\dot{a}^{\mu} \xi_{0}^{\sigma}\right)_{s} D_{b}^{\beta}\left[R_{\mu}(t-s)^{\alpha-1}\right]\right] \mathrm{d} s\right\} \\
& \quad+\left[\left(\frac{\partial R_{v}}{\partial a^{\mu} a} D_{\tau}^{\beta} a^{\nu}-\frac{\partial B}{\partial a^{\mu}}\right)(t-\tau)^{\alpha-1}+{ }_{\tau} D_{b}^{\beta}\left(R_{\mu}(t-\tau)^{\alpha-1}\right)\right]\left(\xi_{\mu}^{\sigma}-\dot{a}^{\mu} \xi_{0}^{\sigma}\right)=-\dot{G}^{\sigma}, \quad(\sigma=1,2, \ldots, r),
\end{aligned}
$$

need to be satisfied. 


\subsection{Fractional Noether Symmetries Based on EEFI}

Definition 3. If the Pfaff action (14) satisfies the equality

$$
\Delta S_{E}=0,
$$

then transformation (6) is said to be Noether symmetric for system (18).
According to Definition 3, using formulas (21) and (23), we have

Criterion 5. If transformation (6) is Noether symmetric, then the equation,

$$
\begin{aligned}
& \left(\frac{\partial R_{\mu}}{\partial a^{\nu} a} D_{\tau}^{\beta} a^{\mu}-\frac{\partial B}{\partial a^{\nu}}\right) \Delta a^{\nu}+\left(\frac{\partial R_{\mu}}{\partial \tau}{ }_{a} D_{\tau}^{\beta} a^{\mu}-\frac{\partial B}{\partial \tau}\right) \Delta \tau+\left(R_{\mu a} D_{\tau}^{\beta} a^{\mu}-B\right) \frac{\mathrm{d}}{\mathrm{d} \tau} \Delta \tau \\
& \quad+R_{\mu}\left({ }_{a} D_{\tau}^{\beta} \Delta a^{\mu}-{ }_{a} D_{\tau}^{\beta}\left(\dot{a}^{\mu} \Delta \tau\right)+{ }_{a} D_{\tau}^{\beta} \dot{a}^{\mu} \Delta \tau\right)+\left(R_{\mu a} D_{\tau}^{\beta} a^{\mu}-B\right) \frac{(\alpha-1) \sin h \tau}{\cosh t-\cos h \tau} \Delta \tau=0
\end{aligned}
$$

needs to be satisfied.

Equation (43) can be written as $r$ equations:

$$
\begin{aligned}
& \left(\frac{\partial R_{\mu}}{\partial a^{\nu}} D_{\tau}^{\beta} a^{\mu}-\frac{\partial B}{\partial a^{\nu}}\right) \xi_{\nu}^{\sigma}+\left(\frac{\partial R_{\mu}}{\partial \tau}{ }_{a} D_{\tau}^{\beta} a^{\mu}-\frac{\partial B}{\partial \tau}\right) \xi_{0}^{\sigma}+\left(R_{\mu a} D_{\tau}^{\beta} a^{\mu}-B\right) \dot{\xi}_{0}^{\sigma} \\
& \quad+R_{\mu}\left({ }_{a} D_{\tau}^{\beta} \xi_{\mu}^{\sigma}-{ }_{a} D_{\tau}^{\beta}\left(\dot{a}^{\mu} \xi_{0}^{\sigma}\right)+{ }_{a} D_{\tau}^{\beta} \dot{a}^{\mu} \xi_{0}^{\sigma}\right)+\left(R_{\mu a} D_{\tau}^{\beta} a^{\mu}-B\right) \frac{(\alpha-1) \sin h \tau}{\cosh t-\cos h \tau} \xi_{0}^{\sigma}=0, \quad(\sigma=1,2, \ldots, r)
\end{aligned}
$$

If $r=1$, equation (44) gives the fractional Noether identity based on EEFI.
Criterion 6. If transformation (7) is Noether symmetric, then the following $r$ equations,

$$
\begin{aligned}
& \frac{\mathrm{d}}{\mathrm{d} \tau}\left\{\left(R_{\mu a} D_{\tau}^{\beta} a^{\mu}-B\right)(\cosh t-\cosh \tau)^{\alpha-1} \xi_{0}^{\sigma}+\int_{a}^{\tau}\left[R_{\mu a} D_{s}^{\beta}\left(\xi_{\mu}^{\sigma}-\dot{a}^{\mu} \xi_{0}^{\sigma}\right)(\cosh t-\cosh \mathrm{s})^{\alpha-1}\right.\right. \\
& \left.\left.\quad-\left(\xi_{\mu}^{\sigma}-\dot{a}^{\mu} \xi_{0}^{\sigma}\right)_{s} D_{b}^{\beta}\left[R_{\mu}(\cosh t-\cosh \mathrm{s})^{\alpha-1}\right]\right] \mathrm{d} s\right\} \\
& \quad+\left[\left(\frac{\partial R_{v}}{\partial a^{\mu} a} D_{\tau}^{\beta} a^{\nu}-\frac{\partial B}{\partial a^{\mu}}\right)(\cosh t-\cosh \tau)^{\alpha-1}+{ }_{\tau} D_{b}^{\beta}\left(R_{\mu}(\cosh t-\cosh \tau)^{\alpha-1}\right)\right]\left(\xi_{\mu}^{\sigma}-\dot{a}^{\mu} \xi_{0}^{\sigma}\right)=0, \quad(\sigma=1,2, \ldots, r)
\end{aligned}
$$

need to be satisfied.

Definition 4. If the Pfaff action (14) satisfies the equality

$$
\Delta S_{E}=-\frac{1}{\Gamma(\alpha)} \int_{a}^{b} \frac{\mathrm{d}}{\mathrm{d} \tau}(\Delta G) \mathrm{d} \tau
$$

where $\Delta G=\varepsilon_{\sigma} G^{\sigma}$ and $G^{\sigma}=G^{\sigma}\left(\tau, a^{\nu}\right)$ is the gauge function, then transformation (6) is said to be Noether quasi-symmetric for system (18).

According to Definition 4, using formulas (21) and (23), we have the following. 
Criterion 7. If transformation (6) is Noether quasi-symmetric, then the equation,

$$
\begin{aligned}
& \left(\frac{\partial R_{\mu}}{\partial a^{\nu} a} D_{\tau}^{\beta} a^{\mu}-\frac{\partial B}{\partial a^{\nu}}\right) \Delta a^{\nu}+\left(\frac{\partial R_{\mu}}{\partial \tau}{ }_{a} D_{\tau}^{\beta} a^{\mu}-\frac{\partial B}{\partial \tau}\right) \Delta \tau \\
& \quad+\left(R_{\mu a} D_{\tau}^{\beta} a^{\mu}-B\right) \frac{d}{d \tau} \Delta \tau+R_{\mu}\left({ }_{a} D_{\tau}^{\beta} \Delta a^{\mu}-{ }_{a} D_{\tau}^{\beta}\left(\dot{a}^{\mu} \Delta \tau\right)+{ }_{a} D_{\tau}^{\beta} \dot{a}^{\mu} \Delta \tau\right) \\
& \quad+\left(R_{\mu a} D_{\tau}^{\beta} a^{\mu}-B\right) \frac{(\alpha-1) \sinh \tau}{\cosh t-\cosh \tau} \Delta \tau=-\frac{d}{d \tau}(\Delta G)(\cosh t-\cosh \tau)^{1-\alpha}
\end{aligned}
$$

needs to be satisfied.

Equation (47) can be written as $r$ equations:

$$
\begin{aligned}
& \left(\frac{\partial R_{\mu}}{\partial a^{\nu} a} D_{\tau}^{\beta} a^{\mu}-\frac{\partial B}{\partial a^{\nu}}\right) \xi_{\nu}^{\sigma}+\left(\frac{\partial R_{\mu}}{\partial \tau}{ }_{a} D_{\tau}^{\beta} a^{\mu}-\frac{\partial B}{\partial \tau}\right) \xi_{0}^{\sigma} \\
& \quad+\left(R_{\mu a} D_{\tau}^{\beta} a^{\mu}-B\right) \dot{\xi}_{0}^{\sigma}+R_{\mu}\left({ }_{a} D_{\tau}^{\beta} \xi_{\mu}^{\sigma}-{ }_{a} D_{\tau}^{\beta}\left(\dot{a}^{\mu} \xi_{0}^{\sigma}\right)+{ }_{a} D_{\tau}^{\beta} \dot{a}^{\mu} \xi_{0}^{\sigma}\right) \\
& \quad+\left(R_{\mu a} D_{\tau}^{\beta} a^{\mu}-B\right) \frac{(\alpha-1) \sinh \tau}{\cosh t-\cosh \tau} \xi_{0}^{\sigma}=-\dot{G}^{\sigma}(\cosh t-\cosh \tau)^{1-\alpha}, \quad(\sigma=1,2, \ldots, r)
\end{aligned}
$$

If $r=1$, equation (48) also gives the fractional Noether identity based on EEFI.
Criterion 8. If transformation (7) is Noether quasi-symmetric, then the following $r$ equations,

$$
\begin{aligned}
& \frac{\mathrm{d}}{\mathrm{d} \tau}\left\{\left(R_{\mu a} D_{\tau}^{\beta} a^{\mu}-B\right)(\cosh t-\cosh \tau)^{\alpha-1} \xi_{0}^{\sigma}+\int_{a}^{\tau}\left[R_{\mu a} D_{s}^{\beta}\left(\xi_{\mu}^{\sigma}-\dot{a}^{\mu} \xi_{0}^{\sigma}\right)(\cosh t-\cosh \mathrm{s})^{\alpha-1}\right.\right. \\
& \left.\left.\quad-\left(\xi_{\mu}^{\sigma}-\dot{a}^{\mu} \xi_{0}^{\sigma}\right)_{s} D_{b}^{\beta}\left[R_{\mu}(\cos h t-\cosh \mathrm{s})^{\alpha-1}\right]\right] \mathrm{d} s\right\} \\
& \quad+\left[\left(\frac{\partial R_{v}}{\partial a^{\mu}} D^{\beta}{ }_{\tau}^{\beta} a^{\nu}-\frac{\partial B}{\partial a^{\mu}}\right)(\cosh t-\cosh \tau)^{\alpha-1}+{ }_{\tau} D_{b}^{\beta}\left(R_{\mu}(\cosh t-\cosh \tau)^{\alpha-1}\right)\right]\left(\xi_{\mu}^{\sigma}-\dot{a}^{\mu} \xi_{0}^{\sigma}\right)=-\dot{G}^{\sigma}, \quad(\sigma=1,2, \ldots, r)
\end{aligned}
$$

need to be satisfied.

\subsection{Fractional Noether Symmetries Based on EPFI}

Definition 5. If the Pfaff action 24 satisfies the equality

$$
\Delta S_{P}=0,
$$

then transformation (6) is said to be Noether symmetric for system (28).

According to Definition 5, using formulas (31) and (33), we have the following.

Criterion 9. If transformation (6) is Noether symmetric, then the equation,

$$
\begin{aligned}
& \left(\frac{\partial R_{\mu}}{\partial a^{\nu}} D^{\beta} a_{\tau}^{\mu}-\frac{\partial B}{\partial a^{\nu}}\right) \Delta a^{\nu}+\left(\frac{\partial R_{\mu}}{\partial \tau}{ }_{a} D_{\tau}^{\beta} a^{\mu}-\frac{\partial B}{\partial \tau}\right) \Delta \tau+\left(R_{\mu a} D_{\tau}^{\beta} a^{\mu}-B\right) \frac{\mathrm{d}}{\mathrm{d} \tau} \Delta \tau \\
& \quad+R_{\mu}\left({ }_{a} D_{\tau}^{\beta} \Delta a^{\mu}-{ }_{a} D_{\tau}^{\beta}\left(\dot{a}^{\mu} \Delta \tau\right)+{ }_{a} D_{\tau}^{\beta} \dot{a}^{\mu} \Delta \tau\right)-\left(R_{\mu a} D_{\tau}^{\beta} a^{\mu}-B\right) \frac{\alpha-1}{\tan ((\alpha-1)(t-\tau)+(\pi / 2))} \Delta \tau=0
\end{aligned}
$$

needs to be satisfied. 
Equation (51) can be written as $r$ equations:

$$
\begin{gathered}
\left(\frac{\partial R_{\mu}}{\partial a^{\nu} a} D_{\tau}^{\beta} a^{\mu}-\frac{\partial B}{\partial a^{\nu}}\right) \xi_{v}^{\sigma}+\left(\frac{\partial R_{\mu}}{\partial \tau}{ }_{a} D_{\tau}^{\beta} a^{\mu}-\frac{\partial B}{\partial \tau}\right) \xi_{0}^{\sigma}+\left(R_{\mu a} D_{\tau}^{\beta} a^{\mu}-B\right) \dot{\xi}_{0}^{\sigma} \\
+R_{\mu}\left({ }_{a} D_{\tau}^{\beta} \xi_{\mu}^{\sigma}-{ }_{a} D_{\tau}^{\beta}\left(\dot{a}^{\mu} \xi_{0}^{\sigma}\right)+{ }_{a} D_{\tau}^{\beta} \dot{a}^{\mu} \xi_{0}^{\sigma}\right)-\left(R_{\mu a} D_{\tau}^{\beta} a^{\mu}-B\right) \\
\frac{\alpha-1}{\tan ((\alpha-1)(t-\tau)+(\pi / 2))} \xi_{0}^{\sigma}=0, \quad(\sigma=1,2, \ldots, r) .
\end{gathered}
$$

If $r=1$, equation (52) gives the fractional Noether identity based on EPFI.
Criterion 10. If transformation (7) is Noether symmetric, then the following $r$ equations,

$$
\begin{aligned}
& \frac{\mathrm{d}}{\mathrm{d} \tau}\left\{\left(R_{\mu a} D_{\tau}^{\beta} a^{\mu}-B\right) \sin \left((\alpha-1)(t-\tau)+\frac{\pi}{2}\right) \xi_{0}^{\sigma}+\int_{a}^{\tau}\left[R_{\mu a} D_{s}^{\beta}\left(\xi_{\mu}^{\sigma}-\dot{a}^{\mu} \xi_{0}^{\sigma}\right) \sin \left((\alpha-1)(t-s)+\frac{\pi}{2}\right)\right.\right. \\
& \left.\left.\quad-\left(\xi_{\mu}^{\sigma}-\dot{a}^{\mu} \xi_{0}^{\sigma}\right)_{s} D_{b}^{\beta}\left[R_{\mu} \sin \left((\alpha-1)(t-s)+\frac{\pi}{2}\right)\right]\right] \mathrm{d} s\right\} \\
& \quad+\left[\left(\frac{\partial R_{v}}{\partial a^{\mu} a} D_{\tau}^{\beta} a^{v}-\frac{\partial B}{\partial a^{\mu}}\right) \sin \left((\alpha-1)(t-\tau)+\frac{\pi}{2}\right)+{ }_{\tau} D_{b}^{\beta}\left(R_{\mu} \sin \left((\alpha-1)(t-\tau)+\frac{\pi}{2}\right)\right)\right]\left(\xi_{\mu}^{\sigma}-\dot{a}^{\mu} \xi_{0}^{\sigma}\right)=0, \quad(\sigma=1,2, \ldots, r),
\end{aligned}
$$

need to be satisfied.

Definition 6. If the Pfaff action (24) satisfies the equality

$$
\Delta S_{P}=-\frac{1}{\Gamma(\alpha)} \int_{a}^{b} \frac{\mathrm{d}}{\mathrm{d} \tau}(\Delta G) \mathrm{d} \tau,
$$

where $\Delta G=\varepsilon_{\sigma} G^{\sigma}$ and $G^{\sigma}=G^{\sigma}\left(\tau, a^{\nu}\right)$ is the gauge function, then transformation (6) is said to be Noether quasi-symmetric for system (28).
According to Definition 6, using formulas (21) and (23), we have the following.

Criterion 11. If transformation (6) is Noether quasi-symmetric, then the equation,

$$
\begin{aligned}
& \left(\frac{\partial R_{\mu}}{\partial a^{\nu}{ }} D_{\tau}^{\beta} a^{\mu}-\frac{\partial B}{\partial a^{\nu}}\right) \Delta a^{\nu}+\left(\frac{\partial R_{\mu}}{\partial \tau}{ }_{a} D_{\tau}^{\beta} a^{\mu}-\frac{\partial B}{\partial \tau}\right) \Delta \tau+\left(R_{\mu a} D_{\tau}^{\beta} a^{\mu}-B\right) \frac{\mathrm{d}}{\mathrm{d} \tau} \Delta \tau \\
& \quad+R_{\mu}\left({ }_{a} D_{\tau}^{\beta} \Delta a^{\mu}-{ }_{a} D_{\tau}^{\beta}\left(\dot{a}^{\mu} \Delta \tau\right)+{ }_{a} D_{\tau}^{\beta} \dot{a}^{\mu} \Delta \tau\right)-\left(R_{\mu a} D_{\tau}^{\beta} a^{\mu}-B\right) \frac{\alpha-1}{\tan ((\alpha-1)(t-\tau)+(\pi / 2))} \\
& \Delta \tau=-\frac{(\mathrm{d} / \mathrm{d} \tau)(\Delta G)}{\sin ((\alpha-1)(t-\tau)+(\pi / 2))}
\end{aligned}
$$

needs to be satisfied. 
Equation (55) can be written as $r$ equations:

$$
\begin{aligned}
& \left(\frac{\partial R_{\mu}}{\partial a^{\nu}} D_{\tau}^{\beta} a^{\mu}-\frac{\partial B}{\partial a^{\nu}}\right) \xi_{\nu}^{\sigma}+\left(\frac{\partial R_{\mu}}{\partial \tau}{ }_{a} D_{\tau}^{\beta} a^{\mu}-\frac{\partial B}{\partial \tau}\right) \xi_{0}^{\sigma}+\left(R_{\mu a} D_{\tau}^{\beta} a^{\mu}-B\right) \dot{\xi}_{0}^{\sigma} \\
& \quad+R_{\mu}\left({ }_{a} D_{\tau}^{\beta} \xi_{\mu}^{\sigma}-{ }_{a} D_{\tau}^{\beta}\left(\dot{a}^{\mu} \xi_{0}^{\sigma}\right)+{ }_{a} D_{\tau}^{\beta} \dot{a}^{\mu} \xi_{0}^{\sigma}\right)-\left(R_{\mu a} D_{\tau}^{\beta} a^{\mu}-B\right) \frac{\alpha-1}{\tan ((\alpha-1)(t-\tau)+(\pi / 2))} \\
& \xi_{0}^{\sigma}=-\frac{\dot{G}^{\sigma}}{\sin ((\alpha-1)(t-\tau)+(\pi / 2))} .
\end{aligned}
$$

If $r=1$, equation (56) gives the fractional Noether identity based on EPFI.
Criterion 12. If transformation (7) is Noether quasi-symmetric, then the following $r$ equations,

$$
\begin{aligned}
& \frac{\mathrm{d}}{\mathrm{d} \tau}\left\{\left(R_{\mu a} D_{\tau}^{\beta} a^{\mu}-B\right) \sin \left((\alpha-1)(t-\tau)+\frac{\pi}{2}\right) \xi_{0}^{\sigma}+\int_{a}^{\tau}\left[R_{\mu a} D_{s}^{\beta}\left(\xi_{\mu}^{\sigma}-\dot{a}^{\mu} \xi_{0}^{\sigma}\right) \sin \left((\alpha-1)(t-s)+\frac{\pi}{2}\right)\right.\right. \\
& \left.\left.\quad-\left(\xi_{\mu}^{\sigma}-\dot{a}^{\mu} \xi_{0}^{\sigma}\right)_{s} D_{b}^{\beta}\left[R_{\mu} \sin \left((\alpha-1)(t-s)+\frac{\pi}{2}\right)\right]\right] \mathrm{d} s\right\} \\
& \quad+\left[\left(\frac{\partial R_{v}}{\partial a^{\mu}} D_{a} D_{\tau}^{\beta} a^{v}-\frac{\partial B}{\partial a^{\mu}}\right) \sin \left((\alpha-1)(t-\tau)+\frac{\pi}{2}\right)+{ }_{\tau} D_{b}^{\beta}\left(R_{\mu} \sin \left((\alpha-1)(t-\tau)+\frac{\pi}{2}\right)\right)\right] \\
& \quad\left(\xi_{\mu}^{\sigma}-\dot{a}^{\mu} \xi_{0}^{\sigma}\right)=-\dot{G}^{\sigma}, \quad(\sigma=1,2, \ldots, r),
\end{aligned}
$$

need to be satisfied.

\subsection{Fractional Noether's Theorems Based on ERLFI}

\section{Fractional Noether's Theorems under Quasi- Fractional Dynamics Models}

Theorem 1. If transformation (7) is Noether symmetric of system (5) based on ERLFI, then

Now, we prove Noether's theorems for fractional Birkhoffian systems under three quasi-fractional dynamics models.

$$
I^{\sigma}=\left(R_{\mu a} D_{\tau}^{\beta} a^{\mu}-B\right)(t-\tau)^{\alpha-1} \xi_{0}^{\sigma}+\int_{a}^{\tau}\left[R_{\mu a} D_{s}^{\beta}\left(\xi_{\mu}^{\sigma}-\dot{a}^{\mu} \xi_{0}^{\sigma}\right)(t-s)^{\alpha-1}-\left(\xi_{\mu}^{\sigma}-\dot{a}^{\mu} \xi_{0}^{\sigma}\right)_{s} D_{b}^{\beta}\left[R_{\mu}(t-s)^{\alpha-1}\right]\right] \mathrm{d} s=c^{\sigma}, \quad(\sigma=1,2, \ldots, r)
$$

are $r$ linearly independent conserved quantities.

Proof. From Definition 1, we get $\Delta S_{R}=0$, namely,

$$
\begin{aligned}
& \frac{1}{\Gamma(\alpha)} \int_{a}^{b} \varepsilon_{\sigma}\left\{\frac{\mathrm{d}}{\mathrm{d} \tau}\left[\left(R_{\mu a} D_{\tau}^{\beta} a^{\mu}-B\right)(t-\tau)^{\alpha-1} \xi_{0}^{\sigma}+\int_{a}^{\tau}\left(R_{\mu a} D_{s}^{\beta}\left(\xi_{\mu}^{\sigma}-\dot{a}^{\mu} \xi_{0}^{\sigma}\right)(t-s)^{\alpha-1}-\left(\xi_{\mu}^{\sigma}-\dot{a}^{\mu} \xi_{0}^{\sigma}\right)_{s} D_{b}^{\beta}\left(R_{\mu}(t-s)^{\alpha-1}\right)\right) \mathrm{d} s\right]\right. \\
& \left.+\left[\left(\frac{\partial R_{v}}{\partial a^{\mu}} D_{\tau}^{\beta} a^{\nu}-\frac{\partial B}{\partial a^{\mu}}\right)(t-\tau)^{\alpha-1}+{ }_{\tau} D_{b}^{\beta}\left(R_{\mu}(t-\tau)^{\alpha-1}\right)\right]\left(\xi_{\mu}^{\sigma}-\dot{a}^{\mu} \xi_{0}^{\sigma}\right) \mathrm{d} \tau\right\}=0 .
\end{aligned}
$$


By substituting (5) into the above formula and considering the arbitrariness of the integral interval and the independence of $\varepsilon_{\sigma}$, we obtain

$$
\frac{\mathrm{d}}{\mathrm{d} \tau}\left[\left(R_{\mu a} D_{\tau}^{\beta} a^{\mu}-B\right)(t-\tau)^{\alpha-1} \xi_{0}^{\sigma}+\int_{a}^{\tau}\left(R_{\mu a} D_{s}^{\beta}\left(\xi_{\mu}^{\sigma}-\dot{a}^{\mu} \xi_{0}^{\sigma}\right)(t-s)^{\alpha-1}-\left(\xi_{\mu}^{\sigma}-\dot{a}^{\mu} \xi_{0}^{\sigma}\right)_{s} D_{b}^{\beta}\left(R_{\mu}(t-s)^{\alpha-1}\right)\right) \mathrm{d} s\right]=0
$$

So, Theorem 1 is proved.

Theorem 2. If transformation (7) is Noether quasi-symmetric of system (5) based on ERLFI, then

$$
\begin{aligned}
I^{\sigma}= & \left(R_{\mu a} D_{\tau}^{\beta} a^{\mu}-B\right)(t-\tau)^{\alpha-1} \xi_{0}^{\sigma}+\int_{a}^{\tau}\left[R_{\mu a} D_{s}^{\beta}\left(\xi_{\mu}^{\sigma}-\dot{a}^{\mu} \xi_{0}^{\sigma}\right)(t-s)^{\alpha-1}-\left(\xi_{\mu}^{\sigma}-\dot{a}^{\mu} \xi_{0}^{\sigma}\right)_{s} D_{b}^{\beta}\left[R_{\mu}(t-s)^{\alpha-1}\right]\right] \mathrm{d} s \\
& +G^{\sigma}=c^{\sigma}, \quad(\sigma=1,2, \ldots, r)
\end{aligned}
$$

are $r$ linearly independent conserved quantities.

Proof. Combining Definition 2 and formula (13), using equation (5), and considering the arbitrariness of the integral interval and the independence of $\varepsilon_{\sigma}$, the conclusion is obtained.

\subsection{Fractional Noether's Theorems Based on EEFI}

Theorem 3. If transformation (7) is Noether symmetric of system (18) based on EEFI, then

$$
\begin{aligned}
I^{\sigma}= & \left(R_{\mu a} D_{\tau}^{\beta} a^{\mu}-B\right)(\cosh t-\cosh \tau)^{\alpha-1} \xi_{0}^{\sigma}+\int_{a}^{\tau}\left[R_{\mu a} D_{s}^{\beta}\left(\xi_{\mu}^{\sigma}-\dot{a}^{\mu} \xi_{0}^{\sigma}\right)(\cosh t-\cosh s)^{\alpha-1}\right. \\
& \left.-\left(\xi_{\mu}^{\sigma}-\dot{a}^{\mu} \xi_{0}^{\sigma}\right)_{s} D_{b}^{\beta}\left[R_{\mu}(\cosh t-\cos h s)^{\alpha-1}\right]\right] \mathrm{d} s=c^{\sigma}, \quad(\sigma=1,2, \ldots, r),
\end{aligned}
$$

are $r$ linearly independent conserved quantities.

Theorem 4. If transformation (7) is Noether quasi-symmetric of system (18) based on EEFI, then

$$
\begin{aligned}
I^{\sigma}= & \left(R_{\mu a} D_{\tau}^{\beta} a^{\mu}-B\right)(\cosh t-\cosh \tau)^{\alpha-1} \xi_{0}^{\sigma}+\int_{a}^{\tau}\left[R_{\mu a} D_{s}^{\beta}\left(\xi_{\mu}^{\sigma}-\dot{a}^{\mu} \xi_{0}^{\sigma}\right)(\cosh t-\cosh s)^{\alpha-1}\right. \\
& \left.-\left(\xi_{\mu}^{\sigma}-\dot{a}^{\mu} \xi_{0}^{\sigma}\right)_{s} D_{b}^{\beta}\left[R_{\mu}(\cosh t-\cosh s)^{\alpha-1}\right]\right] \mathrm{d} s+G^{\sigma}=c^{\sigma}, \quad(\sigma=1,2, \ldots, r),
\end{aligned}
$$

are $r$ linearly independent conserved quantities.

Theorem 5. If transformation (7) is Noether symmetric of system (28) based on EPFI, then

\subsection{Fractional Noether's Theorems Based on EPFI}

$$
\begin{aligned}
I^{\sigma}= & \left(R_{\mu a} D_{\tau}^{\beta} a^{\mu}-B\right) \sin \left((\alpha-1)(t-\tau)+\frac{\pi}{2}\right) \xi_{0}^{\sigma}+\int_{a}^{\tau}\left[R_{\mu a} D_{s}^{\beta}\left(\xi_{\mu}^{\sigma}-\dot{a}^{\mu} \xi_{0}^{\sigma}\right) \sin \left((\alpha-1)(t-s)+\frac{\pi}{2}\right)\right. \\
& \left.-\left(\xi_{\mu}^{\sigma}-\dot{a}^{\mu} \xi_{0}^{\sigma}\right)_{s} D_{b}^{\beta}\left[R_{\mu} \sin \left((\alpha-1)(t-s)+\frac{\pi}{2}\right)\right]\right] \mathrm{d} s=c^{\sigma}, \quad(\sigma=1,2, \ldots, r) .
\end{aligned}
$$

are $r$ linearly independent conserved quantities. 
Theorem 6. If transformation (7) is Noether quasi-symmetric of system (28) based on EPFI, then

$$
\begin{aligned}
I^{\sigma}= & \left(R_{\mu a} D_{\tau}^{\beta} a^{\mu}-B\right) \sin \left((\alpha-1)(t-\tau)+\frac{\pi}{2}\right) \xi_{0}^{\sigma}+\int_{a}^{\tau}\left[R_{\mu a} D_{\tau}^{\beta}\left(\xi_{\mu}^{\sigma}-\dot{a}^{\mu} \xi_{0}^{\sigma}\right) \sin \left((\alpha-1)(t-s)+\frac{\pi}{2}\right)\right. \\
& \left.-\left(\xi_{\mu}^{\sigma}-\dot{a}^{\mu} \xi_{0}^{\sigma}\right)_{s} D_{b}^{\beta}\left[R_{\mu} \sin \left((\alpha-1)(t-s)+\frac{\pi}{2}\right)\right]\right] \mathrm{d} s+G^{\sigma}=c^{\sigma}, \quad(\sigma=1,2, \ldots, r),
\end{aligned}
$$

are $r$ linearly independent conserved quantities.

Obviously, if $\beta \longrightarrow 1$, then Theorems $1-6$ give Noether's theorems for quasi-fractional Birkhoffian systems. If $\alpha \longrightarrow 1$ and $\beta \longrightarrow 1$, Theorems 1-6 give Noether's theorems for classical Birkhoffian systems [58].

\section{Examples}

5.1. Example 1. Consider a fractional Birkhoffian system based on ERLFI. The Pfaff action is

$$
S_{R}=\frac{1}{\Gamma(\alpha)} \int_{a}^{b}\left\{a^{2}{ }_{a} D_{\tau}^{\beta} a^{1}+a^{4}{ }_{a} D_{\tau}^{\beta} a^{3}-a^{2} a^{3}\right\}(t-\tau)^{\alpha-1} d \tau,
$$

where the Birkhoffian is $B=a^{2} a^{3}$, and Birkhoff's functions are $R_{1}=a^{2}, R_{2}=0, R_{3}=a^{4}$, and $R_{4}=0$.

From equation (5), Birkhoff's equations are

$$
\begin{aligned}
{ }_{\tau} D_{b}^{\beta}\left[a^{2}(t-\tau)^{\alpha-1}\right] & =0, \\
{ }_{a} D_{\tau}^{\beta} a^{1}-a^{3} & =0, \\
-a^{2}(t-\tau)^{\alpha-1}+{ }_{\tau} D_{b}^{\beta}\left[a^{4}(t-\tau)^{\alpha-1}\right] & =0, \\
{ }_{a} D_{\tau}^{\beta} a^{3} & =0 .
\end{aligned}
$$

According to (40), the Noether identity gives

$$
\begin{aligned}
& \left({ }_{a} D_{\tau}^{\beta} a^{1}-a^{3}\right) \xi_{2}^{\sigma}-a^{2} \xi_{3}^{\sigma}+{ }_{a} D_{\tau}^{\beta} a^{3} \xi_{4}^{\sigma}+a^{2}\left[{ }_{a} D_{\tau}^{\beta} \xi_{1}^{\sigma}-{ }_{a} D_{\tau}^{\beta}\left(\dot{a}^{1} \xi_{0}^{\sigma}\right)+{ }_{a} D_{\tau}^{\beta} \dot{a}^{1} \xi_{0}^{\sigma}\right] \\
& \quad+a^{4}\left[{ }_{a} D_{\tau}^{\beta} \xi_{3}^{\sigma}-{ }_{a} D_{\tau}^{\beta}\left(\dot{a}^{3} \xi_{0}^{\sigma}\right)+{ }_{a} D_{\tau}^{\beta} \dot{a}^{3} \xi_{0}^{\sigma}\right]+\left[a^{2}{ }_{a} D_{\tau}^{\beta} a^{1}+a^{4}{ }_{a} D_{\tau}^{\beta} a^{3}-a^{2} a^{3}\right] \dot{\xi}_{0}^{\sigma}-\frac{\alpha-1}{t-\tau} \\
& \quad \cdot\left(a^{2}{ }_{a} D_{\tau}^{\beta} a^{1}+a^{4}{ }_{a} D_{\tau}^{\beta} a^{3}-a^{2} a^{3}\right) \xi_{0}^{\sigma}=-\dot{G}^{\sigma}(t-\tau)^{1-\alpha} .
\end{aligned}
$$

Let

$$
\begin{aligned}
& \xi_{0}^{\sigma}=1, \\
& \xi_{1}^{\sigma}=a^{1}, \\
& \xi_{2}^{\sigma}=1, \\
& \xi_{3}^{\sigma}=a^{3}, \\
& \xi_{4}^{\sigma}=1, \\
& G^{\sigma}=0 .
\end{aligned}
$$

By Theorem 2, we obtain

$$
I=\left(a_{a}^{2} D_{\tau}^{\beta} a^{1}+a^{4}{ }_{a} D_{\tau}^{\beta} a^{3}-a^{3} a^{2}\right)(t-\tau)^{\alpha-1}=\text { const. }
$$

The conserved quantity (70) corresponds the Noether symmetry (69).

When $\beta \longrightarrow 1$, formula (70) is reduced to

$$
I=\left(a^{2} a^{1}+a^{4} a^{3}-a^{2} a^{3}\right)(t-\tau)^{\alpha-1}=\text { const. }
$$

Formula (71) is the conserved quantity of Birkhoffian system based on ERLFI.

When $\beta \longrightarrow 1$ and $\alpha \longrightarrow 1$, formula (70) is reduced to

$$
I=a^{2} a^{1}+a^{4} a^{3}-a^{2} a^{3}=\text { const. }
$$

Formula (72) is the classical conserved quantity.

5.2. Example 2. Consider a fractional Birkhoffian system based on EEFI. The Pfaff action is 


$$
S_{E}=\frac{1}{\Gamma(\alpha)} \int_{a}^{b}\left\{\left(a^{2}+a^{3}\right)_{a} D_{\tau}^{\beta} a^{1}+a^{4}{ }_{a} D_{\tau}^{\beta} a^{3}-\left(a^{2} a^{3}+\left(a^{3}\right)^{2}\right)\right\} \cdot(\cosh t-\cosh \tau)^{\alpha-1} \mathrm{~d} \tau
$$

where $\quad B=a^{2} a^{3}+\left(a^{3}\right)^{2}, \quad R_{1}=a^{2}+a^{3}, R_{2}=0, R_{3}=$

From equation (18), Birkhoff's equations are $a^{4}$, and $R_{4}=0$.

$$
\begin{aligned}
{ }_{\tau} D_{b}^{\beta}\left[\left(a^{2}+a^{3}\right)(\cosh t-\cosh \tau)^{\alpha-1}\right] & =0, \\
{ }_{a} D_{\tau}^{\beta} a^{1}-a^{3} & =0, \\
\left({ }_{a} D_{\tau}^{\beta} a^{1}-a^{2}-2 a^{3}\right)(\cosh t-\cosh \tau)^{\alpha-1}+{ }_{\tau} D_{b}^{\beta}\left[a^{4}(\cosh t-\cosh \tau)^{\alpha-1}\right] & =0, \\
{ }_{a} D_{\tau}^{\beta} a^{3} & =0 .
\end{aligned}
$$

According to (48), the Noether identity is

$$
\begin{gathered}
\left({ }_{a} D_{\tau}^{\beta} a^{1}-a^{3}\right) \xi_{2}^{\sigma}+\left({ }_{a} D_{\tau}^{\beta} a^{1}-a^{2}-2 a^{3}\right) \xi_{3}^{\sigma}+{ }_{a} D_{\tau}^{\beta} a^{3} \xi_{4}^{\sigma}+\left(a^{2}+a^{3}\right)\left[{ }_{a} D_{\tau}^{\beta} \xi_{1}^{\sigma}-{ }_{a} D_{\tau}^{\beta}\left(\dot{a}^{1} \xi_{0}^{\sigma}\right)+{ }_{a} D_{\tau}^{\beta} \dot{a}^{1} \xi_{0}^{\sigma}\right] \\
+a^{4}\left[{ }_{a} D_{\tau}^{\beta} \xi_{3}^{\sigma}-{ }_{a} D_{\tau}^{\beta}\left(\dot{a}^{3} \xi_{0}^{\sigma}\right)+{ }_{a} D_{\tau}^{\beta} \dot{a}^{3} \xi_{0}^{\sigma}\right]+\left[\left(a^{2}+a^{3}\right)_{a} D_{\tau}^{\beta} a^{1}+a^{4}{ }_{a} D_{\tau}^{\beta} a^{3}-a^{2} a^{3}-\left(a^{3}\right)^{2}\right] \dot{\xi}_{0}^{\sigma} \\
+\frac{(\alpha-1) \sin h \tau}{\cosh t-\cosh \tau}\left(\left(a^{2}+a^{3}\right)_{a} D_{\tau}^{\beta} a^{1}+a^{4}{ }_{a} D_{\tau}^{\beta} a^{3}-a^{2} a^{3}-\left(a^{3}\right)^{2}\right) \xi_{0}^{\sigma}=-\dot{G}^{\sigma}(\cos h t-\cos h \tau)^{1-\alpha} .
\end{gathered}
$$

Let

$$
\begin{aligned}
& \xi_{0}=1, \\
& \xi_{1}=a^{1}, \\
& \xi_{2}=0, \\
& \xi_{3}=a^{3}, \\
& \xi_{4}=0, \\
& G^{\sigma}=0 .
\end{aligned}
$$

By Theorem 3, we obtain

$$
\begin{aligned}
I= & {\left[\left(a^{2}+a^{3}\right)_{a} D_{\tau}^{\beta} a^{1}+a^{4}{ }_{a} D_{\tau}^{\beta} a^{3}-a^{2} a^{3}-\left(a^{3}\right)^{2}\right] } \\
& \cdot(\cosh t-\cosh \tau)^{\alpha-1}=\text { const. }
\end{aligned}
$$

The conserved quantity (77) corresponds to the Noether symmetry (76).
If $\beta \longrightarrow 1$, then we obtain

$$
\begin{aligned}
I= & {\left[\left(a^{2}+a^{3}\right) a^{1}+a^{4} a^{3}-a^{2} a^{3}-\left(a^{3}\right)^{2}\right] } \\
& \cdot(\cosh t-\cosh \tau)^{\alpha-1}=\text { const. }
\end{aligned}
$$

Formula (78) is the conserved quantity of Birkhoffian system based on EEFI.

If $\beta \longrightarrow 1$ and $\alpha \longrightarrow 1$, then we obtain

$$
I=\left(a^{2}+a^{3}\right) a^{1}+a^{4} a^{3}-a^{2} a^{3}-\left(a^{3}\right)^{2}=\text { const. }
$$

Formula (79) is the classical conserved quantity.

5.3. Example 3. Consider a fractional Birkhoffian system based on EPFI. The Pfaff action is

$$
S_{P}=\frac{1}{\Gamma(\alpha)} \int_{a}^{b}\left\{\left[a^{3}{ }_{a} D_{\tau}^{\beta} a^{1}+a_{a}^{4} D_{\tau}^{\beta} a^{2}-\frac{1}{2}\left(a^{3}\right)^{2}-\frac{1}{2}\left(a^{4}\right)^{2}\right] \cdot \sin \left((\alpha-1)(t-\tau)+\frac{\pi}{2}\right)\right\} \mathrm{d} \tau,
$$

where $\quad B=(1 / 2)\left(a^{3}\right)^{2}+(1 / 2)\left(a^{4}\right)^{2}, \quad R_{1}=a^{3}, R_{2}=$ $a^{4}$, and $R_{3}=R_{4}=0$. 
From equation (28), Birkhoff's equations are

$$
\begin{aligned}
{ }_{\tau} D_{b}^{\beta}\left[a^{3} \sin \left((\alpha-1)(t-\tau)+\frac{\pi}{2}\right)\right] & =0, \\
{ }_{\tau} D_{b}^{\beta}\left[a^{4} \sin \left((\alpha-1)(t-\tau)+\frac{\pi}{2}\right)\right] & =0, \\
{ }_{a} D_{\tau}^{\beta} a^{1}-a^{3} & =0, \\
{ }_{a} D_{\tau}^{\beta} a^{2}-a^{4} & =0 .
\end{aligned}
$$

According to (56), the Noether identity is

$$
\begin{aligned}
\left({ }_{a} D_{\tau}^{\beta} a^{1}-a^{3}\right) \xi_{3}+\left({ }_{a} D_{\tau}^{\beta} a^{2}-a^{4}\right) \xi_{4}+a^{3}\left({ }_{a} D_{\tau}^{\beta} \xi_{1}^{\sigma}-{ }_{a} D_{\tau}^{\beta}\left(\dot{a}^{1} \xi_{0}^{\sigma}\right)+{ }_{a} D_{\tau}^{\beta} \dot{a}^{1} \xi_{0}^{\sigma}\right) \\
\quad+a^{4}\left({ }_{a} D_{\tau}^{\beta} \xi_{2}^{\sigma}-{ }_{a} D_{\tau}^{\beta}\left(\dot{a}^{2} \xi_{0}^{\sigma}\right)+{ }_{a} D_{\tau}^{\beta} \dot{a}^{2} \xi_{0}^{\sigma}\right)+\left(a^{3}{ }_{a} D_{\tau}^{\beta} a^{1}+a^{4}{ }_{a} D_{\tau}^{\beta} a^{2}-\left(\frac{1}{2}\left(a^{3}\right)^{2}+\frac{1}{2}\left(a^{4}\right)^{2}\right)\right) \dot{\xi}_{0}^{\sigma} \\
\quad-\frac{\alpha-1}{\tan ((\alpha-1)(t-\tau)+(\pi / 2))}\left(a^{3}{ }_{a} D_{\tau}^{\beta} a^{1}+a^{4}{ }_{a} D_{\tau}^{\beta} a^{2}-\left(\frac{1}{2}\left(a^{3}\right)^{2}+\frac{1}{2}\left(a^{4}\right)^{2}\right)\right) \xi_{0}^{\sigma} \\
=-\dot{G}^{\sigma} \frac{1}{\sin ((\alpha-1)(t-\tau)+(\pi / 2))}
\end{aligned}
$$

Let

$$
\begin{aligned}
\xi_{0} & =0, \\
\xi_{1} & =a^{2}, \\
\xi_{2} & =-a^{1}, \\
\xi_{3} & =\xi_{4}=0, \\
G^{\sigma} & =0 .
\end{aligned}
$$

By Theorem 5, we obtain

$$
\begin{aligned}
I= & \int_{a}^{\tau}\left\{a^{3}{ }_{a} D_{\tau}^{\beta} a^{2} \sin \left((\alpha-1)(t-s)+\frac{\pi}{2}\right)\right\} \\
& -a^{4}{ }_{a} D_{\tau}^{\beta} a^{1} \sin \left((\alpha-1)(t-s)+\frac{\pi}{2}\right) \\
& -a^{2}{ }_{s} D_{b}^{\beta}\left[a^{3} \sin \left((\alpha-1)(t-s)+\frac{\pi}{2}\right)\right] \\
& \left.+a^{1}{ }_{s} D_{b}^{\beta}\left[a^{4} \sin \left((\alpha-1)(t-s)+\frac{\pi}{2}\right)\right]\right\} \mathrm{d} s \\
= & \text { const. }
\end{aligned}
$$

The conserved quantity (84) corresponds to the Noether symmetry (83).

When $\beta \longrightarrow 1$, formula (84) becomes

$$
I=\left(a^{3} a^{2}-a^{4} a^{1}\right) \sin \left((\alpha-1)(t-\tau)+\frac{\pi}{2}\right)=\text { const. }
$$

Formula (85) is the conserved quantity of Birkhoffian system based on EPFI.

When $\beta \longrightarrow 1$ and $\alpha \longrightarrow 1$, formula (84) becomes

$$
I=a^{3} a^{2}-a^{4} a^{1}=\text { const. }
$$

Formula (86) is the classical conserved quantity.

\section{Conclusions}

By introducing fractional calculus into the dynamic modeling of nonconservative systems, the dynamic behavior and physical process of complex systems can be described more accurately, which provides the possibility for the quantization of nonconservative problems. Compared with fractional models, the quasi-fractional model greatly simplifies the calculation of complex fractional-order calculus, so it can be used to study complex nonconservative dynamic systems more conveniently. The dynamics of Birkhoffian system is an extension of Hamiltonian mechanics, and the fractional Birkhoffian system is an extension of integer Birkhoffian system. Therefore, fractional Birkhoffian dynamics is a research field worthy of further study and full of vitality.

The main contributions of this paper are as follows. Firstly, based on three quasi-fractional dynamics models, the fractional Pfaff-Birkhoff principles and fractional Birkhoff's equations are established, in which the Pfaff action contains fractionalorder derivative terms. Secondly, the fractional Noether symmetry is explored, and its definitions and criteria are 
established. Thirdly, Noether's theorems for fractional Birkhoffian systems under three quasi-fractional dynamics models are proved, and fractional conservation laws are obtained.

Obviously, the results of the following two systems are special cases of this paper: (1) the quasi-fractional Birkhoffian systems based on quasi-fractional dynamics models, in which the Pfaff action contains only integer-order derivative terms; (2) the classical Birkhoffian systems under integer-order models. Therefore, our study is of great significance.

\section{Data Availability}

The data used to support the findings of this study are included within the article.

\section{Conflicts of Interest}

The author declares that there are no conflicts of interest regarding the publication of this paper.

\section{Acknowledgments}

This work was supported by the National Natural Science Foundation of China (nos. 11972241, 11572212, and 11272227) and Natural Science Foundation of Jiangsu Province of China (no. BK20191454).

\section{References}

[1] A. E. Noether, "Invariante variationsprobleme," Nachr Akad Wiss Gottingen Math-Phys, vol. 2, pp. 235-257, 1918.

[2] E. Candotti, C. Palmieri, and B. Vitale, "On the inversion of noether's theorem in classical dynamical systems," American Journal of Physics, vol. 40, no. 3, pp. 424-429, 1972.

[3] E. A. Desloge and R. I. Karch, "Noether's theorem in classical mechanics," American Journal of Physics, vol. 45, no. 4, pp. 336-339, 1977.

[4] D. S. Vujanovic and B. D. Vujanović, "Noether's theory in classical nonconservative mechanics," Acta Mechanica, vol. 23, no. 1-2, pp. 17-27, 1975.

[5] D. Liu, "Noether theorem and its inverse for nonholonomic conservative dynamical systems," Science in China Series A, vol. 20, no. 11, pp. 1189-1197, 1991.

[6] M. Lutzky, "Dynamical symmetries and conserved quantities," Journal of Physics A: Mathematical and General, vol. 12, no. 7, pp. 973-981, 1979.

[7] P. J. Olver, Applications of Lie Groups to Differential Equations, Springer-Verlag, New York, NY, USA, 1986.

[8] N. H. Ibragimov, CRC Handbook of Lie Group Analysis of Differential Equations, CRC Press, Boca Raton, FL, USA, 1994.

[9] G. W. Bluman and S. Kumei, Symmetries and Differential Equations, Springer-Verlag, New York, NY, USA, 1989.

[10] Y. Y. Zhao, "Conservative quantities and Lie symmetries of nonconservative dynamical systems," Acta Mechanica Sinica, vol. 26, pp. 380-384, 1994.

[11] F. X. Mei, Applications of Lie Groups and Lie Algebras to Constrained Mechanical Systems, Science Press, Beijing, China, 1999.

[12] F. X. Mei, Symmetries and Conserved Quantities of Constrained Mechanical Systems, Beijing Institute of Technology, Beijing, China, 2004.
[13] Y. Zhang, "Noether's theorem for a time-delayed Birkhoffian system of Herglotz type," International Journal of Non-linear Mechanics, vol. 101, pp. 36-43, 2018.

[14] L. J. Zhang and Y. Zhang, "Non-standard Birkhoffian dynamics and its Noether's theorems," Communications in Nonlinear Science and Numerical Simulation, vol. 91, p. 11, Article ID 105435, 2020.

[15] C. J. Song and Y. Chen, "Noether's theorems for nonshifted dynamic systems on time scales," Applied Mathematics and Computation, vol. 374, p. 12, Article ID 125086, 2020.

[16] H.-B. Zhang and H.-B. Chen, "Noether's theorem of Hamiltonian systems with generalized fractional derivative operators," International Journal of Non-linear Mechanics, vol. 107, pp. 34-41, 2018.

[17] Y. Zhang, "Herglotz's variational problem for nonconservative system with delayed arguments under Lagrangian framework and its Noether's theorem," Symmetry, vol. 12, no. 5, p. 13, Article ID 845, 2020.

[18] S. X. Jin and Y. Zhang, "Noether theorem for nonholonomic systems with time delay," Mathematical Problems in Engineering, vol. 2015, p. 9, Article ID 539276, 2015.

[19] P.-P. Cai, J.-L. Fu, and Y.-X. Guo, "Lie symmetries and conserved quantities of the constraint mechanical systems on time scales," Reports on Mathematical Physics, vol. 79, no. 3, pp. 279-298, 2017.

[20] M. J. Lazo, J. Paiva, and G. S. F. Frederico, "Noether theorem for action-dependent Lagrangian functions: conservation laws for non-conservative systems," Nonlinear Dynamics, vol. 97, no. 2, pp. 1125-1136, 2019.

[21] Y. Zhang, "Lie symmetry and invariants for a generalized Birkhoffian system on time scales," Chaos, Solitons \& Fractals, vol. 128, pp. 306-312, 2019.

[22] F. X. Mei, H. B. Wu, and Y. F. Zhang, "Symmetries and conserved quantities of constrained mechanical systems," International Journal of Dynamics and Control, vol. 2, no. 3, pp. 285-303, 2014.

[23] M. Inc, A. Yusuf, A. I. Aliyu, and D. Baleanu, "Lie symmetry analysis, explicit solutions and conservation laws for the space-time fractional nonlinear evolution equations," Physica A: Statistical Mechanics and Its Applications, vol. 496, pp. 371-383, 2018.

[24] D. Baleanu, M. Inc, A. Yusuf, and A. I. Aliyu, "Lie symmetry analysis, exact solutions and conservation laws for the time fractional Caudrey-Dodd-Gibbon-Sawada-Kotera equation," Communications in Nonlinear Science and Numerical Simulation, vol. 59, pp. 222-234, 2018.

[25] K. B. Oldham and J. Spanier, The Fractional Calculus, Academic Press, San Diego, CL, USA, 1974.

[26] K. S. Miller and B. Ross, An Introduction to the Fractional Integrals and Derivatives-Theory and Applications, John Wiley \& Sons, New York, NY, USA, 1993.

[27] I. Podlubny, Fractional Differential Equations, Academic Press, San Diego, CL, USA, 1999.

[28] A. A. Kilbas, H. M. Srivastava, and J. J. Trujillo, Theory and Applications of Fractional Differential Equations, Elsevier B V, Amsterdam, The Netherlands, 2006.

[29] R. Hilfer, Applications of Fractional Calculus in Physics, World Scientific, Singapore, 2000.

[30] R. Herrmann, Fractional Calculus: An Introduction for Physicists, World Scientific Publishing, Singapore, 2014.

[31] V. E. Tarasov, "Fractional diffusion equations for open quantum system," Nonlinear Dynamics, vol. 71, no. 4, pp. 663-670, 2013. 
[32] R. E. Gutiérrez, J. M. Rosário, and J. T. Machado, "Fractional order calculus: basic concepts and engineering applications," Mathematical Problems in Engineering, vol. 2010, Article ID 375858, 19 pages, 2010.

[33] J. A. T. Machado, M. F. Silva, R. S. Barbosa et al., "Some applications of fractional calculus in engineering," Mathematical Problems in Engineering, vol. 2010, Article ID 639801, 34 pages, 2010.

[34] D. Baleanu, J. H. Asad, and I. Petras, "Fractional batemanfeshbach tikochinsky oscillator," Communications in Theoretical Physics, vol. 61, no. 2, pp. 221-225, 2014.

[35] Y. Zhang, "Fractional differential equations of motion in terms of combined Riemann-Liouville derivative," Chinese Physics B, vol. 21, no. 8, 5 pages, Article ID 084502, 2012.

[36] F. Riewe, "Nonconservative Lagrangian and Hamiltonian mechanics," Physical Review E, vol. 53, no. 2, pp. 1890-1899, 1996.

[37] F. Riewe, "Mechanics with fractional derivatives," Physical Review E, vol. 55, no. 3, pp. 3581-3592, 1997.

[38] R. A. El-Nabulsi, "A fractional approach to nonconservative Lagrangian dynamical systems," Fizika A, vol. 14, no. 4, pp. 289-298, 2005.

[39] R. A. El-Nabulsi, "Fractional variational problems from extended exponentially fractional integral," Computers \& Mathematics with Applications, vol. 217, no. 22, pp. 94929496, 2011.

[40] R. A. El-Nabulsi, "A periodic functional approach to the calculus of variations and the problem of time-dependent damped harmonic oscillators," Appled Mathematics Letters, vol. 24, no. 10, pp. 1647-1653, 2011.

[41] G. S. F. Frederico and D. F. M. Torres, "A formulation of Noether's theorem for fractional problems of the calculus of variations," Journal of Mathematical Analysis and Applications, vol. 334, no. 2, pp. 834-846, 2007.

[42] T. M. Atanacković, S. Konjik, S. Pilipović, and S. Simić, "Variational problems with fractional derivatives: invariance conditions and Noether's theorem," Nonlinear Analysis, vol. 71, no. 5-6, pp. 1504-1517, 2009.

[43] A. B. Malinowska and D. F. M. Torres, Introduction to the Fractional Calculus of Variations, Imperial College Press, London, UK, 2012.

[44] R. Almeida, S. Pooseh, and D. F. M. Torres, Computational Methods in the Fractional Calculus of Variations, Imperial College Press, Singapore, 2015.

[45] J. Cresson and A. Szafrańska, “About the Noether's theorem for fractional Lagrangian systems and a generalization of the classical Jost method of proof," Fractional Calculus and Applied Analysis, vol. 22, no. 4, pp. 871-898, 2019.

[46] X. Tian and Y. Zhang, "Noether's theorem for fractional Herglotz variational principle in phase space," Chaos, Solitons \& Fractals, vol. 119, pp. 50-54, 2019.

[47] X. Tian and Y. Zhang, "Fractional time-scales Noether theorem with Caputo $\Delta$ derivatives for Hamiltonian systems," Applied Mathematics and Computation, vol. 393, Article ID 125753, 15 pages, 2021.

[48] S. Zhou, H. Fu, and J. Fu, "Symmetry theories of Hamiltonian systems with fractional derivatives," Science China Physics, Mechanics and Astronomy, vol. 54, no. 10, pp. 1847-1853, 2011.

[49] C. J. Song, "Noether symmetry for fractional Hamiltonian system," Physics Letters A, vol. 383, Article ID 125914, 2019.

[50] G. S. F. Frederico and D. F. M. Torres, "Constants of motion for fractional action-like variational problems," International
Journal of Applied Mathematics, vol. 19, no. 1, pp. 97-104, 2006.

[51] G. S. F. Frederico and D. F. M. Torres, "Nonconservative Noether's theorem for fractional action-like variational problems with intrinsic and observer times," International Journal of Ecological Economics and Statistics, vol. 91, pp. 74-82, 2007.

[52] Y. Zhang and Y. Zhou, "Symmetries and conserved quantities for fractional action-like Pfaffian variational problems," Nonlinear Dynamics, vol. 73, no. 1-2, pp. 783-793, 2013.

[53] Z.-X. Long and Y. Zhang, "Fractional Noether theorem based on extended exponentially fractional integral," International Journal of Theoretical Physics, vol. 53, no. 3, pp. 841-855, 2014.

[54] Z.-X. Long and Y. Zhang, "Noether's theorem for fractional variational problem from El-Nabulsi extended exponentially fractional integral in phase space," Acta Mechanica, vol. 225, no. 1, pp. 77-90, 2014.

[55] Z. X. Long and Y. Zhang, "Noether's theorem for nonconservative Hamilton system based on El-Nabulsi dynamical model extended by periodic laws," Chinese Physics B, vol. 23, no. 11, 9 pages, Article ID 114501, 2014.

[56] G. D. Birkhoff, Dynamical Systems, AMS College Publisher, Providence, RI, USA, 1927.

[57] R. M. Santilli, Foundations of Theoretical Mechanics II, Springer, New York, NY, USA, 1983.

[58] F. X. Mei, R. C. Shi, Y. Zhang, and H. B. Wu, Dynamics of Birkhoffian Systems, Beijing Institute of Technology, Beijing, China, 1996.

[59] Y. Zhang and X.-H. Zhai, "Noether symmetries and conserved quantities for fractional Birkhoffian systems," Nonlinear Dynamics, vol. 81, no. 1-2, pp. 469-480, 2015.

[60] X.-H. Zhai and Y. Zhang, "Noether symmetries and conserved quantities for fractional Birkhoffian systems with time delay," Communications in Nonlinear Science and Numerical Simulation, vol. 36, no. 1-2, pp. 81-97, 2016.

[61] Y. Zhou and Y. Zhang, "Noether theorems of a fractional Birkhoffian system within Riemann-Liouville derivatives," Chinese Physics B, vol. 23, no. 12, Article ID 124502, 2014.

[62] C.-J. Song and Y. Zhang, "Noether symmetry and conserved quantity for fractional Birkhoffian mechanics and its applications," Fractional Calculus and Applied Analysis, vol. 21, no. 2, pp. 509-526, 2018.

[63] Q. Jia, H. Wu, and F. Mei, "Noether symmetries and conserved quantities for fractional forced Birkhoffian systems," Journal of Mathematical Analysis and Applications, vol. 442, no. 2, pp. 782-795, 2016.

[64] H.-B. Zhang and H.-B. Chen, "Noether's theorem of fractional Birkhoffian systems," Journal of Mathematical Analysis and Applications, vol. 456, no. 2, pp. 1442-1456, 2017.

[65] X. Tian and Y. Zhang, "Noether symmetry and conserved quantities of fractional Birkhoffian system in terms of Herglotz variational problem," Communications in Theoretical Physics, vol. 70, no. 3, pp. 280-288, 2018.

[66] C. J. Song, "Adiabatic invariants for generalized fractional Birkhoffian mechanics and their applications," Mathematical Problems in Engineering, vol. 2018, Article ID 6414960, 18 pages, 2018. 